\title{
Combined Photothermotherapy and Chemotherapy of Oral Squamous Cell Carcinoma Guided by Multifunctional Nanomaterials Enhanced Photoacoustic Tomography
}

\author{
Sujuan Zeng ${ }^{1} *$ \\ Shiqi Liu ${ }^{1,2, *}$ \\ Yintao Lan $^{3}$ \\ Ting Qiu ${ }^{3}$ \\ Mengyu Zhou ${ }^{3}$ \\ Weijian $\mathrm{Gao}^{3}$ \\ Wenyan Huang' \\ Lihong Ge $\mathrm{G}^{1,4}$ \\ Jian Zhang (D) ${ }^{1,3}$
}

'Department of Pedodontics, Affiliated Stomatology Hospital of Guangzhou Medical University, Guangzhou Key Laboratory of Basic and Applied Research of Oral Regenerative Medicine, Guangdong, People's Republic of China;

${ }^{2}$ Department of Stomatology, Taihe Hospital, Institute of Oral Diseases, School of Dentistry, Hubei University of Medicine, Hubei, People's Republic of China; ${ }^{3}$ Department of Biomedical Engineering, School of Basic Medical Sciences, The Sixth Affiliated Hospital of Guangzhou Medical University, Qingyuan People's Hospital, Guangzhou Medical University, Guangdong, People's Republic of China; ${ }^{4}$ Department of Pediatric Dentistry, Stomatology Hospital of Peking University, Beijing, People's Republic of China

*These authors contributed equally to this work
Background: Squamous cell carcinoma of the head and neck is the sixth most common cancer worldwide, with $40 \%$ occurring in the oral cavity. Although the level of early diagnosis and treatment of OSCC has improved significantly, the five-year survival rate of advanced patients remains unsatisfactory. However, the main challenges before us are how to get an early and accurate diagnosis and how to formulate effective treatment. Nanoparticlebased chemo-photothermal therapy has proven to be a promising non-invasive approach to treating oral squamous cell carcinoma treatment.

Methods: In this study, we tried to design and synthesize multifunctional hyaluronic acid (HA) modified gold nanorods/mesoporous silica-based nanoparticles loaded with doxorubicin hydrochloride (DOX) for photoacoustic imaging (PAI) guided cooperative chemophotothermal therapy.

Results: The resultant nanocomposite shows favorable biocompatibility, relatively low cytotoxicity, ideal drug loading capability and strong PAI signals. In addition, they showed an excellent photothermal conversion efficiency of $49.02 \%$ for photothermal therapy (PTT). Moreover, in vivo and in vitro experiments have shown that synergistic chemo-photothermal therapy has better therapeutic effects than chemotherapy alone or PTT $(P<0.05)$. After being injected into the CAL-27 tumor-bearing mice, the DOX-AuNRs@ $\mathrm{mSiO}_{2}-\mathrm{HA}$ nanoparticles could accumulate rapidly at the tumor sites and achieve complete ablation of tumors when combined with near-infrared laser irradiation, without obvious side effects on normal tissues.

Conclusion: Our research provides a solid demonstration of the potential of DOX-AuNRs@mSiO ${ }_{2}-\mathrm{HA}$ as a multifunctional platform in PAI-guided photothermal chemotherapy for oral squamous cell carcinoma.

Keywords: mesoporous silica coated gold nanorods, photoacoustic imaging, chemophotothermal therapy, oral squamous cell carcinoma

\section{Introduction}

Up to now, cancer continues to be the world's malignant disease and the leading cause of death. ${ }^{1-3}$ Head and neck cancer is the sixth largest malignant tumor worldwide, of which $40 \%$ occur in the oral cavity and nearly $90 \%$ of which is classified as oral squamous cell carcinoma (OSCC). ${ }^{4}$ For early stage cancers, radiotherapy and surgery can effectively play a role. Whereas advanced cancers typically require combinations of surgery, chemotherapy and radiotherapy. ${ }^{5}$ OSCC
Correspondence: Jian Zhang; Lihong Ge Email jianzhang@gzhmu.edu.cn; gelh0919@126.com 
is preventable and curable at an early stage, but the majority of cases can only be diagnosed at an advanced stage. Moreover, advanced therapy is difficult to achieve rational effect and its prognosis is terrible. ${ }^{6,7}$ The 5-year survival rate of advanced OSCC patients is still unsatisfactory, which is mainly related to the early diagnosis difficulty, easy recurrence after treatment, large systemic adverse effects and so on, ${ }^{8-10}$ making OSCC one of the most challenging diseases and remain a global health concern regardless of several advances made in the field of OSCC diagnostics and therapeutics. ${ }^{11}$

Currently, precision medicine and targeted therapy are increasingly popular with anticancer treatments. ${ }^{12}$ With the rapid development of modern imaging technology, photoacoustic imaging (PAI) is an emerging non-ionizing and non-invasive diagnostic technique, which enables tissue visualization with high resolution and in-depth resolution. ${ }^{13-15}$ Relatively recently, the use of PAI in the field of the oral cavity has been widely reported. In the diagnosis and assessment of the curative effect of OSCC, PAI plays an important role, especially in the localization of tumor areas and the detection of metastatic lymph nodes. ${ }^{16-18}$ Another conspicuous characteristic of PAI is that it offers the possibility of combining diagnosis to treatments, such as photothermal therapy (PTT). ${ }^{19-22}$ PTT is a therapeutic method that uses targeted recognition technology to gather materials with excellent photothermal conversion efficiency near tumor tissue, and converts light energy into heat energy to kill cancer cells under the irradiation of near-infrared light. ${ }^{23-25}$ Consequently, as a promising substitute for effective cancer treatment, it has attracted considerable attention. Owing to physiological complexity of serious diseases such as cancer, current developments have gently shifted from a focus on monotherapy to combine or multiple therapies since the synergy of therapeutic agents or techniques give rise to ostentatious super additive (namely " $1+1>2$ ") therapeutic effects. ${ }^{26}$

Cancer nanomedicine is considered to be a relatively new interdisciplinary field of research, involving chemistry, engineering, drug delivery and medicine, aimed at promoting greater development of cancer diagnosis and treatment. ${ }^{27-30}$ Gold nanoparticles (Au nanoparticles) are commonly used nanoparticles, which have been widely employed in PAI, PTT and targeted drug delivery because of its simple synthesis method, high yield, stable chemical properties, and adjustable surface plasmon resonance. ${ }^{31-36}$ Nevertheless, the low loading capacity of anticancer drugs prevented $\mathrm{Au}$ nanoparticles being used for clinical purposes. Mesoporous silica nanoparticles, as a drug delivery system of high specific surface area, large pore volume, easy surface modification, good biocompatibility and convenient drug loading, have been widely used to coat gold nanorods in order to improve the long-term stability, hydrophilicity and drug delivery ability. ${ }^{37-40}$ Even more importantly, the mesoporous silica coating is a good way to overcome the toxicity of metal nanoparticles, because the silica shell has biochemical stability and corrosion resistance under extreme conditions. ${ }^{41-43}$

In cancer treatment, targeted anticancer drugs can be more effective than conventional chemotherapy. ${ }^{44}$ Hyaluronic acid (HA) is a natural, biodegradable and non-immunogenic polymer with high affinity for CD44 receptor, which has been proven to be involved in various of tumor biological activities including tumor proliferation, progression and metastasis. ${ }^{45,46}$ The HA shell wraps the drug to play a protective role, enhances the stability of the drug, and achieves sustained release of the drug. ${ }^{47,48}$ Herein, mesoporous silica-coated gold nanoparticles with a large volume of pores have been successfully obtained, which can be used for the chemophotothermal synergistic therapy of tumor. Excellent photothermal conversion efficiency and high-resolution photoacoustic (PA) signal were obtained simultaneously by the synthesized gold nanomaterials system. Meanwhile, doxorubicin hydrochloride (DOX), an effective anti-tumor chemotherapy drug, was loaded into the mesoporous silica of AuNRs@ $\mathrm{mSiO}_{2}$ through electrostatic interaction, namely van der Waals forces. ${ }^{49-51}$ HA molecules were coupled to AuNRs@ $\mathrm{mSiO}_{2}$ nanoparticles via amide bonds. To sum up, our DOX-AuNRs@mSiO $\mathrm{m}_{2}$-HA nanoparticles have shown excellent prospects as an effective platform for tumor targeting and PAI-guided chemo-photothermal synergistic therapy for oral cancer.

\section{Experimental Details}

\section{Materials}

Sodium borohydride $\left(\mathrm{NaBH}_{4}\right), \mathrm{HAuCl}_{4}$ Chloroauric acid trihydrate $\left(\mathrm{HAuCl}_{4} \cdot 3 \mathrm{H}_{2} \mathrm{O}\right)$, Cetyltrimethylammonium bromide (CTAB), silver nitrate $\left(\mathrm{AgNO}_{3}\right)$, ascorbic acid, tetraethyl orthosilicate (TEOS) and 3-Aminopropyltriethoxysilane (APTES) were purchased from Shanghai Sigma-Aldrich. DOX and HA were obtained from Ruixi Biological Technology Co., Ltd. Cell Counting Kit-8 (CCK-8) was purchased from Dojindo Laboratories (Japan). A LIVE/DEAD Viability/Cytotoxicity Kit was obtained from the Shanghai Bestbio Biotechnology Co. Ltd. DAPI was purchased from 
Shanghai Beyotime Biotechnology Co. Ltd. The human tongue squamous cell carcinoma CAL-27 cell line and the human oral keratinocytes $\mathrm{HOK}$ cell line were obtained from the American Type Culture Collection (ATCC). Deionised water (Millipore Milli-Q grade) with resistivity of $18.2 \mathrm{M} \Omega$ was used in all the experiments.

\section{Synthesis of AuNRs Nanoparticles}

Depending on the published program, AuNRs were synthesized and some minor modifications were made. ${ }^{52}$ Firstly, CTAB-capped Au seeds were established by chemical reduction of $\mathrm{HAuCl}_{4}$ with $\mathrm{NaBH}_{4}$. $7.5 \mathrm{~mL} 0.1$ $\mathrm{M}$ CTAB was mixed with $250 \mu \mathrm{L} 0.01 \mathrm{M} \mathrm{HAuCl}_{4}$ and the volume was fixed on $9.4 \mathrm{~mL}$ by adding water. Next, $0.6 \mathrm{~mL}$ of ice-cold $\mathrm{NaBH}_{4}$ aqueous solution $(0.01 \mathrm{M})$ was added to the above mentioned mixed solution to form nano-gold seeds, which were stirred for $2 \mathrm{~min}$ and placed at $30^{\circ} \mathrm{C}$ for $2 \mathrm{~h}$. The seeds were immediately formed and used within $2 \sim 5 \mathrm{~h}$. The growth solution for owners consists of $200 \mathrm{~mL}$ of $0.1 \mathrm{M} \mathrm{CTAB}, 10 \mathrm{~mL}$ of 0.01 $\mathrm{M} \mathrm{HAuCl}_{4}, 0.8 \mathrm{~mL}$ of $10 \mathrm{~mm} \mathrm{AgNO}_{3}, 4 \mathrm{~mL}$ of 0.5 $\mathrm{M} \mathrm{H}_{2} \mathrm{SO}_{4}$ and $1.6 \mu \mathrm{L}$ of $0.1 \mathrm{M}$ ascorbic acid. After adding $500 \mu \mathrm{L}$ of the above managed seeds, stirring for two minutes and then standing at $30^{\circ} \mathrm{C}$ overnight. Then, the nanoparticles were collected by centrifugation, and washed twice with deionized water at a speed of $8000 \mathrm{rpm}$ for $20 \mathrm{~min}$ each time. Finally, the owners were dispersed in $250 \mathrm{~mL}$ of deionized water.

\section{Synthesis of AuNRs@mSiO Nanoparticles}

Add a certain amount of CTAB $(0.8 \mathrm{~mm})$ to $250 \mathrm{~mL}$ AuNRs obtained above, and $\mathrm{pH}$ was up-regulated in 10 with the $0.1 \mathrm{~m} \mathrm{NaOH}$ solution, then stirred at $33^{\circ} \mathrm{C}$ for half an hour to mix well. Following this step, $750 \mu \mathrm{L}$ of $20 \%$ TEOS reagent diluted with methanol was added to the owner's solution three times with an interval of half an hour. After the reaction for $24 \mathrm{~h}$, the obtained owners were harvested by centrifugation $(12,000 \mathrm{rpm}, 30 \mathrm{~min})$. Washing with water and ethanol twice, and finally dispersing in $20 \mathrm{~mL}$ of ethanol.

\section{Synthesis of AuNRs@mSiO $2-\mathrm{NH}_{2}$ Nanoparticles}

To prepare amino-modified nanoparticles (AuNRs@ $\mathrm{mSiO}_{2}-\mathrm{NH}_{2}$ ), $50 \mu \mathrm{L}$ of 3-aminopropyltriethoxysilane (APTES) was added to the above-mentioned
AuNRs@ $\mathrm{mSiO}_{2}$ ethanol solution at $60^{\circ} \mathrm{C}$ for 24 h. Subsequently, it was centrifuged and washed three times with ethanol. To remove the surfactant CTAB remaining in the pores, AuNRs@ $\mathrm{mSiO}_{2}-\mathrm{NH}_{2}$ was dispersed in $20 \mathrm{~mL}$ of ammonium nitrate ethanol solution $\left(8 \mathrm{mg} \mathrm{mL}^{-1}\right)$, refluxed overnight, and then centrifuged and washed twice.

\section{Synthesis of DOX-AuNRs@mSiO 2 Nanoparticles}

The amount of DOX loaded into the AuNRs@mSiO $\mathrm{miH}_{2}-\mathrm{NH}_{2}$ nanoparticles was determined by UV-Vis spectrophot-

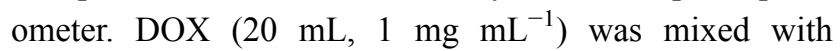
AuNRs@ $\mathrm{mSiO}_{2}-\mathrm{NH}_{2} \quad$ nanoparticles $\quad(16 \quad \mathrm{~mL}$, $2.5 \mathrm{mg} \mathrm{mL}^{-1}$ ), and stirred at $30^{\circ} \mathrm{C}$ for $24 \mathrm{~h}$. After centrifugation, DOX-loaded AuNRs@ $\mathrm{mSiO}_{2}-\mathrm{NH}_{2}$ nanoparticles were obtained and washed with deionized water three times to remove the DOX adsorbed on the surface. The free DOX in the supernatant was determined by UV-Vis spectrophotometer at $480 \mathrm{~nm}$. The DOX-loading efficiency (LE) was calculated by Eq.1:

$$
\begin{aligned}
\mathrm{LE}(\%)= & \frac{\text { WinitialDOX }- \text { WremanentDOX }}{\text { WAuNRs@mSi02 }- \text { NH2 }} \\
& \times 100 \%\left(m g \cdot m g^{-1}\right)
\end{aligned}
$$

\section{Synthesis of DOX-AuNRs@mSiO $2-H A$ Nanoparticles}

At first, $20 \mathrm{mg}$ of HA was dispersed in $15 \mathrm{~mL}$ of deionized water and hydrated for $24 \mathrm{~h}$, and then $8 \mathrm{mg}$ of EDC and $5 \mathrm{mg}$ of the NHS was mixed and stirred for $4 \mathrm{~h}$ to activate the carboxyl group of HA. Finally, DOX-AuNRs@mSiO was added to the HA solution, stirred for $24 \mathrm{~h}$, and the final product could be obtained after centrifugal washing.

\section{Characterization}

The morphology and size of the prepared nanoparticles were characterized by transmission electron microscopy (TEM) (JEM 1400, JEOM, Japan). According to the instructions in the manufacturer's operating manual, use the zeta potential analyzer (Malvern, UK) to detect the zeta potential distribution. The absorption spectra of free Dox, AuNRs, AuNRs@mSiO $\mathrm{m}_{2}-\mathrm{NH}_{2}, \quad$ DOXAuNRs@mSiO 2 , DOX-AuNRs@mSiO ${ }_{2}-\mathrm{HA}$ was acquired using a visible spectrometer (UV-2600, Shimadzu, Japan). Laser-confocal scanning imaging was obtained using a Laser-confocal scanning imaging (Zeiss LSM780, Oberkochen, Germany). Photoacoustic

(PA) 
characterization was conducted with a homemade multispectral PA microscopy (MSPAM) system.

\section{Drug Release Experiments}

Three portions of the prepared DOX-AuNRs@ $\mathrm{mSiO}_{2}-\mathrm{HA}$ nanoparticles at equal amount were dispersed at $\mathrm{pH}=5.0$ PBS $(1.0 \mathrm{~mL})$ and transferred protracted semipermeable dialysis bags at $37^{\circ} \mathrm{C}$. The amount of DOX released into the solution was determined by measuring the absorbance at $480 \mathrm{~nm}$ with a UV-Vis spectrophotometer at selected time intervals. In order to prove that the laser irradiation and hyaluronidase treatment can induce the drug release, other experiments were conducted under the same procedures. Immerse the sample in PBS with near infrared region (NIR) irradiation $\left(808 \mathrm{~nm}, 2.0 \mathrm{~W} \mathrm{~cm}^{-2}\right.$ ) for 5 min, or dissolved it on PBS containing hyaluronidase. The concentration of DOX in the supernatant was evaluated by UV-Vis spectrophotometer.

\section{Photothermal Effect Experiments}

The photothermal effect of AuNRs@ $\mathrm{mSiO}_{2}$-HA nanoparticles was studied by $808 \mathrm{~nm}$ laser. The $808 \mathrm{~nm}$ laser equipment with adjustable power output $\left(0.5-2 \mathrm{~W} \mathrm{~cm}^{-2}\right)$ was used as the light source, and AuNRs@ $@ \mathrm{mSiO}_{2}$-HA nanoparticles $\left(0-200 \mu \mathrm{g} \mathrm{mL}{ }^{-1}\right)$ with different concentrations were dispersed in an EP tube. Use a digital thermometer to monitor the real-time changes of temperature every $10 \mathrm{~s}$. Meanwhile, AuNRs@mSiO ${ }_{2}$-HA was irradiated under 808 nm laser for $600 \mathrm{~s}$, and then naturally cooled for $600 \mathrm{~s}$ to evaluate the photo-stability. The entire process was repeated five times. In addition, the temperature variation of the AuNRs@ $\mathrm{mSiO}_{2}$ -HA nanoparticles was recorded under $808 \mathrm{~nm}$ laser irradiation $\left(2.0 \mathrm{~W} \mathrm{~cm}^{-2}\right)$ until a steady temperature was reached. Photothermal conversion efficiency $(\eta)$ was calculated by Roper's method.

\section{In vitro PAI Experiments}

The PA performance of the AuNRs@ $@ \mathrm{mSiO}_{2}$-HA nanoparticles was evaluated by our MSPAM system. In our MSPAM system (Figure S1), the energy source for exciting materials was a nanosecond pulse OPO laser with tunable wavelength (Continuum, USA), and an ultrasonic transducer with a center frequency of $25 \mathrm{MHz}$ (Olympus, USA) was used to detect the generated acoustic signal. A piece of copper wire was soaked in an agar made solid model and further imaged by our MSPAM system. As shown in Figure S2, the deepest depth of the copper wire that could be imaged can reach $2.3 \mathrm{~mm}$. This result verifies the imaging depth of our MSPAM system. In addition, the full width at half maxima (FWHM) of the line-spread function (LSF) defining the lateral resolution of the PAI system is $37.6 \mu \mathrm{m}$ (Figure S3). To test the linearity of the PA signal as a function of AuNRs@ $@ \mathrm{mSiO}_{2}$-HA nanoparticles, $0.2 \mathrm{~mL}$ of the AuNRs@ $\mathrm{mSiO}_{2}$-HA nanoparticles aqueous suspension with different $\mathrm{Au}$ concentrations ( 0 , $0.2,0.4,0.8$ and $1.0 \mathrm{mg} \mathrm{mL}^{-1}$ ) were added to the agarphantom container and evaluated by our MSPAM system. ${ }^{53,54}$ A complete PA image of the phantom was collected at $808 \mathrm{~nm}$.

\section{Cell Culture}

The human tongue squamous cell carcinoma CAL-27 cell line was selected for in vitro and in vivo experiment in this study. The human oral keratinocytes cell line was chosen for in vitro experiment in this study. The HOK cells were cultured in DMEM medium supplemented with $10 \%$ fetal bovine serum (FBS) in the humidified incubator $\left(37^{\circ} \mathrm{C}\right.$, 5\% $\mathrm{CO}_{2}$ ). The HOK cells were cultured in DMEM: F-12 medium supplemented with $10 \%$ fetal bovine serum (FBS) and $1 \%$ penicillin and streptomycin in the humidified incubator $\left(37^{\circ} \mathrm{C}, 5 \% \mathrm{CO}_{2}\right)$.

\section{Cellular Uptake Experiments}

To investigate the uptake ability of DOX-AuNRs@ $\mathrm{mSiO}_{2}$ HA nanoparticles, CAL-27 and HOK cells were seeded in the 12 -well plate for $24 \mathrm{~h}$, with $2 \times 10^{5}$ cells each well. Then, the culture medium was removed, and DOXAuNRs@mSiO $\mathrm{m}_{2}$ nanoparticles, DOX-AuNRs@ $\mathrm{mSiO}_{2}$ HA nanoparticles were added into the well for 2, 4, 8, and $12 \mathrm{~h}$. The fluorescence intensity of cells was measured by flow cytometry to investigate the correlation of uptake of nanoparticle concentration and incubation time. Similarly, the CAL-27 cells were seeded on 35-mm glass bottomed dished for $24 \mathrm{~h}$. Then, DOX-AuNRs@mSiO $2_{2}$ HA nanoparticles were added into the dishes and incubated for $2 \mathrm{~h}, 4 \mathrm{~h}$ and $8 \mathrm{~h}$, respectively. After that, the cells were stained with DAPI. After 20 min incubation, cells were washed three times with PBS and then fixed with $4 \%$ paraformaldehyde for $30 \mathrm{~min}$ at room temperature. Finally, cells were viewed under the confocal microscope.

\section{In vitro Cytotoxicity Evaluation Against HOK Cells and CAL-27 Cells}

The cytotoxicity of empty AuNRs@mSiO $\mathrm{m}_{2}$-HA nanoparticles, free DOX and DOX-loaded AuNRs@mSiO 2 - $\mathrm{HA}$ 
nanoparticles were evaluated by Cell Counting Kit-8. HOK cells were seeded in a 96-well plate at a density of $3 \times 10^{4}$ per well and incubated in the atmosphere of $95 \%$ air and $5 \% \mathrm{CO}_{2}$ at $37^{\circ} \mathrm{C}$ with $10 \%$ fetal bovine serum and $1 \%$ penicillin and streptomycin for $24 \mathrm{~h}$. Cal-27 cells were seeded in a 96-well plate at a density of $5 \times 10^{4}$ per well and incubated in the atmosphere of $95 \%$ air and $5 \% \mathrm{CO}_{2}$ at $37^{\circ} \mathrm{C}$ with $10 \%$ fetal bovine serum for $24 \mathrm{~h}$. Then, various concentrations of empty AuNRs@mSiO2-HA nanoparticles, free DOX and DOX-loaded AuNRs@ $\mathrm{mSiO}_{2}-\mathrm{HA}$ nanoparticles were added, respectively. In another plate, the suspensions of AuNRs@mSiO $\mathrm{m}_{2}$-HA nanoparticles and DOX-AuNRs@mSiO 2 -HA nanoparticles with different concentrations were added into CAL-27 cells with 808$\mathrm{nm}$ NIR laser irradiation for $5 \mathrm{~min}$. One row of a 96-well plate was added culture medium only to be a blank control. Ten microliters of the CCK- 8 reagent was added to each well and then incubated for $2 \mathrm{~h}$ and the OD value at $450 \mathrm{~nm}$ was read using the micro plate reader. Cell viability was calculated by the percentage of OD value of the study group over the control group.

\section{Live/Dead Cell Viability Assay}

CAL-27 cells were cultured in the 48-well plate for $24 \mathrm{~h}$, DOX-AuNRs@mSiO $\mathrm{m}_{2}$-HA suspension was added into dishes co-incubated for another $6 \mathrm{~h}$. Then CAL-27 cells were irradiated under $808 \mathrm{~nm}$ laser for 5 mins. Afterwards, cells were stained with casein acetomethoxyl (calcein$\mathrm{AM}$ ) and propidium iodide (PI) for $15 \mathrm{~min}$. Fluorescence images of cells were captured by a fluorescence microscope. The $\lambda_{\text {ex }} / \lambda_{\text {em }}$ of calcein-AM and PI were $499 / 515 \mathrm{~nm}$ and $495 / 635 \mathrm{~nm}$, respectively.

\section{Animal and Tumor Model}

Female BALB/c nude mice (4-6 weeks old) were purchased from Guangdong Medical Laboratory Animal Center Co., Ltd. All animal procedures were performed in accordance with the Guidelines for Institutional Animal Care and Use Committee and approved by the approval of the Animal Care and Usage Committee of Guangzhou Medical University (GY2019-009). A human OSCC xenograft tumor model of mice was established by injecting $0.1 \mathrm{~mL}$ CAL-27 cells suspension $\left(4 \times 10^{6}\right)$ into the right flank of the female BALB/c nude mice.

The mice were used for subsequent experiments until the tumor size reached approximately $80 \mathrm{~mm}^{3}$, and the tumor volume was calculated according to the following equation: tumor volume $\left(\mathrm{mm}^{3}\right)=0.5 \times$ (tumor length) $\times$ (tumor width). ${ }^{2}$ The xenograft-bearing mice were randomly assigned into four groups: control group (injected with PBS), LASER group, DOX-AuNRs@mSiO $\mathrm{m}_{2}-\mathrm{HA}$ group (injection with $0.2 \mathrm{~mL}$ DOX-AuNRs@mSiO $\mathrm{m}_{2}-\mathrm{HA}$, $1 \mathrm{mg} \mathrm{mL} \mathrm{mL}^{-1}$, based on gold ion), and DOXAuNRs@mSiO 2 -HA + LASER group (injection with 0.2 mL DOX-AuNRs@ $\mathrm{mSiO}_{2}-\mathrm{HA}, 1 \mathrm{mg} \mathrm{mL}{ }^{-1}$, based on gold ion). The nanoparticles and PBS were intravenously injected via tail vein. ${ }^{55}$ Herein, PTT treatment groups were subjected to NIR laser irradiation (power density of laser, $2 \mathrm{~W} \mathrm{~cm}^{-2}$ ) for $5 \mathrm{~min}$ at $8 \mathrm{~h}$ post injection.

\section{In vivo PAI Experiments}

To evaluate the in vivo photoacoustic performance of the DOX-AuNRs@mSiO 2 -HA for the CAL-27 subcutaneous tumor, the CAL-27 tumor-bearing nude mice were intravenously injected with the as-prepared nanoparticles (200 $\mu \mathrm{L}, 1 \mathrm{mg} \mathrm{mL}^{-1}$ ). For PAI, tumor-bearing mice with intravenously injected nanoparticles aqueous solution were anesthetized (with 1\% sodium pentobarbital). PA images of the tumor site were obtained at different time intervals $(0,2,4,8,12$ and $24 \mathrm{~h})$ and captured by our MSPAM system with $845 \mathrm{~nm}$ laser excitation.

\section{Histological Examinations}

For histological examination, the tissues and tumors of mice were harvested immediately after the mice were sacrificed. After that, 4\% paraformaldehyde solution was used to fix for hematoxylin and eosin (H\&E) staining. All of the sections were examined with an inverted light microscope.

\section{Statistical Analysis}

Data were analyzed with an analysis of variance (one-way ANOVA) using GraphPad Prism 9 software to evaluate the differences between test groups. Statistical significance was defined as $p<0.05$. All data were presented as mean \pm standard deviation (SD). All figures presented in this article were obtained from five independent experiments with similar results.

\section{Result and Discussion}

Fabrication of DOX-AuNRs@mSiO $\mathrm{m}_{2}$-HA nanoparticles is elucidated in Figure 1. Firstly, we constructed AuNRs coated with mesoporous silica nanoparticles. Then, the anticancer drug DOX was loaded into the AuNRs@ $\mathrm{mSiO}_{2}$ nanoparticles via electrostatic interaction. Finally, the amine functioned AuNRs@ $\mathrm{mSiO}_{2}$ by the post 


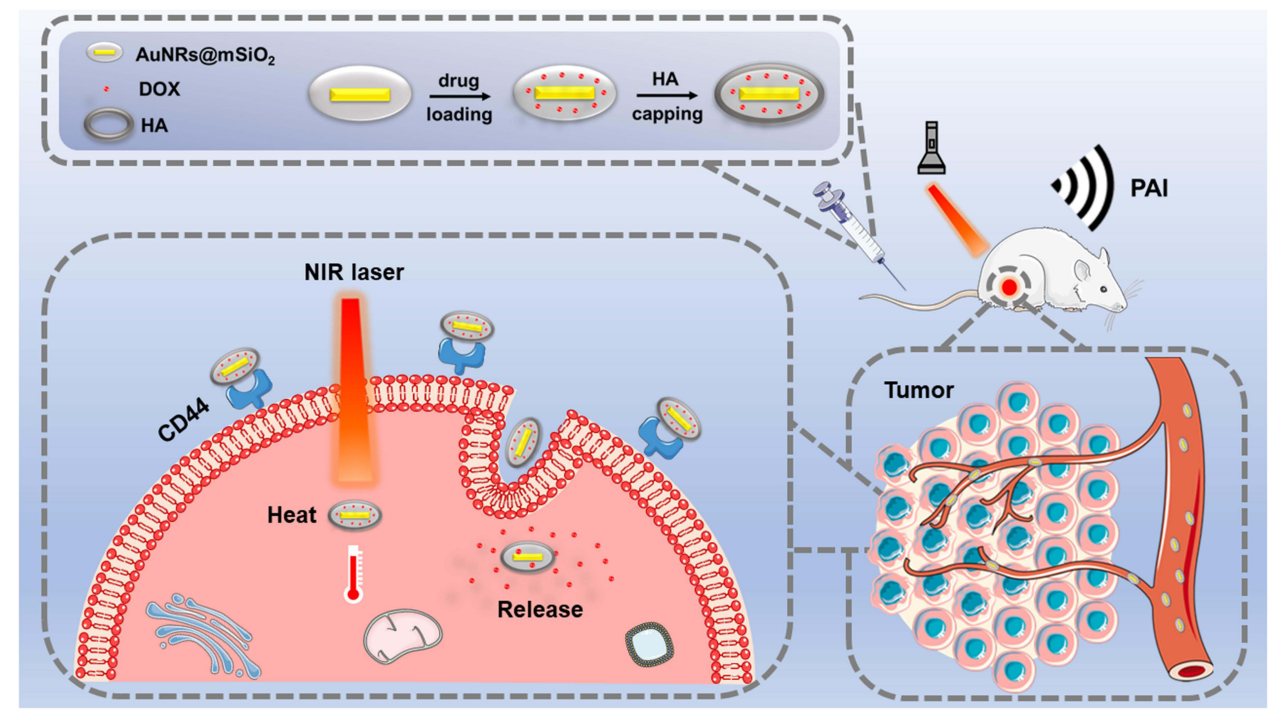

Figure I Schematic illustration of the preparation of HA-modified owners coated mesoporous silica nanoparticles as a carrier of DOX for photoacoustic imaging (PAI) and photothermal-chemo therapy of tumors.

grafting method was attached to HA through the EDCmediated amidation reaction, forming DOXAuNRs@mSiO 2 -HA nanoparticles for photothermal and chemotherapy applications. Meanwhile, CD44 receptorspecific endocytosis of nanoparticles was also demonstrated in Figure 1. When DOX-AuNRs@mSiO $2-H A$ nanoparticles have been injected into a tumor model, the conjugated HA molecules could make DOXAuNRs@mSiO 2 -HA specifically deliver in cancer cells by the CD44-HA. Subsequently, HA shell was able to be degraded by hyaluronidase, which triggered the release of DOX. Under NIR irradiation, the PAI and photothermalchemotherapy of DOX-AuNRs@ $\mathrm{mSiO}_{2}$-HA nanoparticles could be triggered, which led to a synergistic effect for killing cancer cells. In this study, we hypothesized that the drug loaded AuNRs@mSiO ${ }_{2}$ would effectively reach the tumor site by the EPR effect and by the specific CD44 binding on tumor cells. Once bound to $\mathrm{CD} 44$, the nanoparticles would be internalized by receptor-mediated endocytosis, hopefully leading to rapid degradation of the nanoparticles by Hyal-1 and the burst release of the anticancer drug for enhanced therapeutic efficacy.

\section{Synthesis and Characterization of DOX- AuNRs@mSiO 2 -HA}

The preparation of AuNRs was carried out by a seedmediated growth method that has been considered as a simple and cost-effective method of preparation for nanocomposite. ${ }^{56}$ The morphology and size of asprepared AuNRs was characterized by the TEM, which was displayed in Figure 2A. The TEM image showed that the as-prepared AuNRs was about rod shape and highly uniform size. The aspect ratio of high-quality AuNRs was 3.8 (average width, $18 \pm 3 \mathrm{~nm}$; length, $75 \pm 8 \mathrm{~nm}$ ). As shown in Figure S4A, the prepared owners have a weak transverse plasmon band at $510 \mathrm{~nm}$ and a strong longitudinal plasmon band at $750 \mathrm{~nm}$. However, AuNRs required further modifications as drug-delivery platforms. Mesoporous silica nanoparticles have been widely accepted to coat AuNRs to achieve long-term stability, hydrophilicity, and drug delivery capacity. ${ }^{57}$ As illustrated in Figure 2B, the TEM image of the prepared AuNRs@mSiO 2 core-shell nanoparticles showed that the owners were coated with mesoporous silica shells (16 nm thickness), offering an opportunity for AuNRs@ $\mathrm{SiO}_{2}$ to be used as a general drug carrier. However, due to both the increase in the refractive index of the surrounding medium and the changes in nanoparticles geometric, ${ }^{58,59}$ the longitudinal surface plasmon resonance peak exhibited a small red-shift $(40 \mathrm{~nm})$ after silica coating (Figures $\underline{\mathrm{S} 4 \mathrm{~B}}$ and 2E). After the addition of DOX and HA, the shell of DOX-AuNRs@ $\mathrm{mSiO}_{2}$-HA was slightly thicker than that AuNRs@mSiO ${ }_{2}$ due to HA coating (Figure 2C). Once the DOX was absorbed on the surface of nanocomposite, the absorption spectrum of DOX-AuNRs@ $\mathrm{mSiO}_{2}$ had a small peak near $480 \mathrm{~nm}$, locating at a similar place to that of free DOX (Figure 2D). In addition, as depicted 

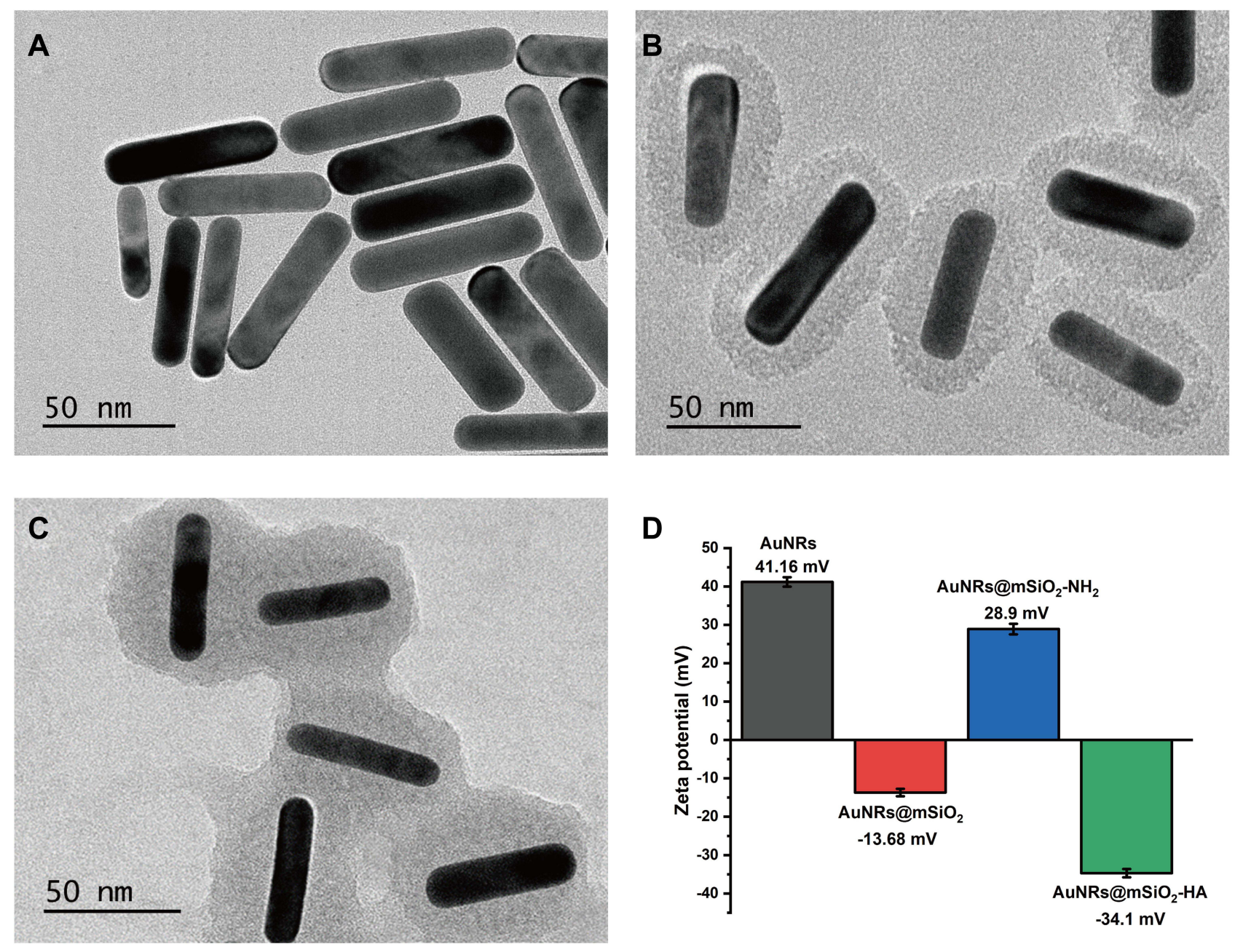

D
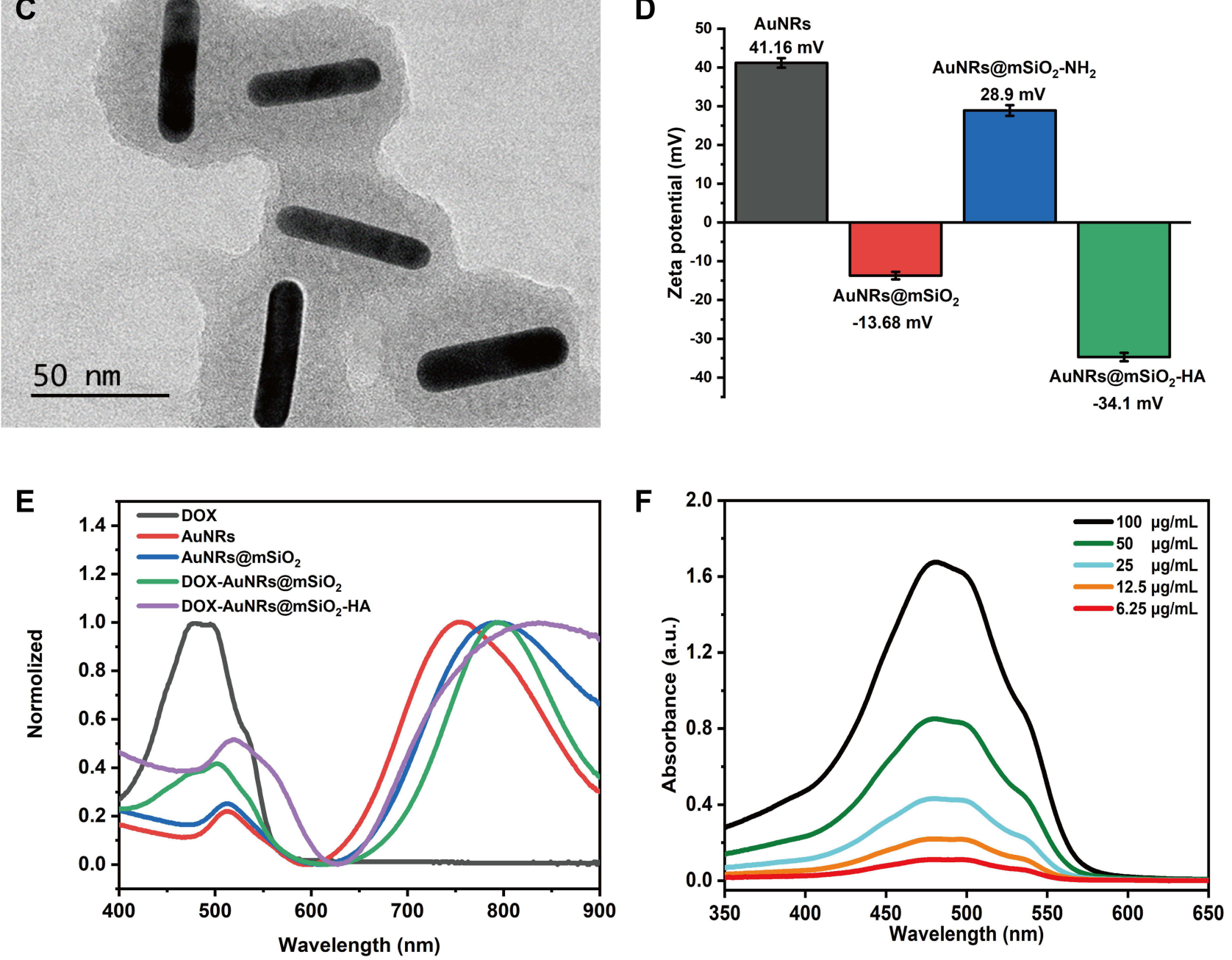

Figure 2 Characterizations of nanocomposites. (A) TEM image of GNRs. Inset scale bar = $50 \mathrm{~nm}$. (B) TEM image of AuNRs@mSiO 2. Inset scale bar = 50nm. (C) TEM images of DOX-AuNRs@mSiO $-\mathrm{HA}$. Inset scale bar=50 nm. (D) Zeta potential distributions of AuNRs, AuNRs@mSiO, $\mathrm{AuNRs} @ \mathrm{mSiO}_{2}-\mathrm{NH}_{2}$, and AuNRs@mSiO $\mathrm{Au}_{2}-\mathrm{HA}$. (E) Absorbance spectrum of DOX, aunts, AuNRs@mSiO, DOX-AuNRs@mSiO 2 , and DOX-AuNRs@mSiO $-\mathrm{HA}$. (F) Absorbance spectrum change of DOX. 
in Figure 2E, a further red-shift accompanying slight damping of the band was observed upon DOX adsorption. All results demonstrated that DOX was successfully loaded on the surface of $\mathrm{mSiO}_{2}$-capped AuNRs-based nanocarrier. The surface charge of the nanoparticles was monitored by a dynamic light scattering instrument (Figure 2F). The surface charge of AuNRs was $+41.16 \mathrm{mg}$ since CTAB molecules were served as player surfactants. After attachment of the mesoporous silica, the potential was decreased to $-13.68 \mathrm{mV}$ because the mesoporous silica itself was negatively charged, and the potential rose to $+28.9 \mathrm{mV}$ due to its positive charge when connected to $-\mathrm{NH}_{2}$. After grafting HA via covalent conjugation to the surface of AuNRs@mSiO tial was $-34.1 \mathrm{MV}$. This effect suggests that the AuNRs@mSiO $\mathrm{m}_{2}$ has been successfully modified by HA. As shown in Figure S5, no aggregation phenomenon was found after storage in the PBS and blood environment at different time points, and it remained dark brown, which led to the stability of DOX-AuNRs@mSiO $\mathrm{mSA}_{2}$ nanoparticles.

\section{In vitro Photothermal Effect}

Considering that the owners possess absorption coefficients, the photothermal efficiency of AuNRs-based nanocomposites was further evaluated in vitro by an $808 \mathrm{~nm}$ diode laser. As shown in Figure 3A, the photothermal conversion efficiency of AuNRs@ $\mathrm{mSiO}_{2}-\mathrm{HA}$ at different concentrations was assessed under the $808 \mathrm{~nm}$ laser irradiation for $5 \mathrm{~min}\left(2 \mathrm{~W} \mathrm{~cm}{ }^{-2}\right)$. The temperature of the AuNRs@ $\mathrm{mSiO}_{2}-\mathrm{HA}$ aqueous suspension rose rapidly with the prolonged laser irradiation time or the increasing concentration of AuNRs@ $\mathrm{mSiO}_{2}-\mathrm{HA}$ aqueous (Figure

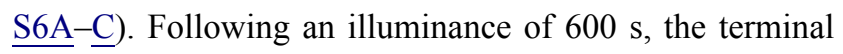
temperature of the AuNRs@ $\mathrm{mSiO}_{2}$-HA aqueous suspension was increased by $26.3^{\circ} \mathrm{C}$ at a concentration of 200 $\mu \mathrm{g} \mathrm{mL}{ }^{-1}$. In the meantime, the temperature increment of pure water is negligible, which can be visually testified by infrared thermal images of as-prepared products (Figure 3C). Furthermore, the photothermal conversion performance of the rod-based AuNRs@mSiO 2 -HA was obviously dependent on the laser-power (Figure 3B). The AuNRs@mSiO $\mathrm{m}_{2}$-HA exhibited excellent chemical stability and stable photothermal conversion performance. The stability of the photothermal effect was evaluated through five laser on/off cycles. As shown in Figures 3D and $\underline{\mathrm{S}} 6 \mathrm{D}$, the temperature of the AuNRs@mSiO $\mathrm{m}_{2}-\mathrm{HA}$ aqueous suspension $\left(100 \mu \mathrm{g} \mathrm{mL}^{-1}, 200 \mu \mathrm{g} \mathrm{mL}^{-1}\right)$ could still reach the similar level after repeated NIR laser irradiation for five cycles, indicating the attractive photothermal stability and re-usability of AuNRs@ $\mathrm{mSiO}_{2}-\mathrm{HA}$ during a longrange laser exposure. Photothermal conversion efficiency $(\eta)$ is a crucial parameter used to evaluate the photothermal agents. On the basis of the heating-cooling curve in Figure 3E, the photothermal conversion efficiency of AuNRs@ $\mathrm{mSiO}_{2}$-HA was calculated to be $49.02 \%$ via the quantification method by Roper, ${ }^{60}$ which was higher than the previously reported photothermal conversion nanomaterials, including $\mathrm{Ag}_{2} \mathrm{~S}$ nanoparticles (28.35\%), ${ }^{61}$ gold nanoclusters $(30.0 \%){ }^{62}$ gold nanocages $(41.7 \%)^{63}$ and so on. Due to its outstanding photothermal conversion efficiency and photothermal stability, AuNRs@mSiO 2 -HA with high photothermal efficiency has great PTT potential at mild temperatures.

\section{DOX Release from DOX- AuNRs@mSiO $2-\mathrm{HA}$}

A standard curve of DOX was drawn (Figure S7) and the sample concentration was determined based on the standard DOX curve. The loading capacity of the AuNRs@ $\mathrm{mSiO}_{2}$ was evaluated by measuring the DOX concentration in solution before and after loading into the nanoparticles using UVVis spectrophotometer at $480 \mathrm{~nm}$, and the drug loading capability of DOX-AuNRs@ $\mathrm{mSiO}_{2}$-HA was $8.0 \% \pm 0.9 \%$. The DOX release behavior of DOX-AuNRs@ $\mathrm{mSiO}_{2}-\mathrm{HA}$ nanoparticles was further evaluated. As shown in Figure $3 \mathrm{~F}$, approximately $24.3 \%$ of DOX released from DOX-AuNRs@mSiO 2 -HA nanoparticles over 24 h. This result indicated a high affinity and subsequent tight retention of the surface of DOX-AuNRs@ $\mathrm{mSiO}_{2}$-HA nanoparticles toward DOX molecules, which is beneficial for preventing premature release and result in sustained in vivo therapeutic effect. To further understand the influence of NIR radiation on DOX release performance, DOX-AuNRs@mSiO $\mathrm{m}_{2}-\mathrm{HA}$ nanoparticles dispersion was irradiated with $808 \mathrm{~nm}$ laser ( $2 \mathrm{~W} \mathrm{~cm}^{-2}, 5 \mathrm{~min}$ ). As shown in Figure 3F, nearly $62.3 \%$ of DOX molecules were released from DOX-AuNRs@ $\mathrm{mSiO}_{2}-$ HA nanoparticles eventually. The burst release of DOX was mainly due to the rapid temperature increasing of AuNRs@ $\mathrm{mSiO}_{2}$ dispersion under NIR irradiation, which facilitates the DOX molecule thermal motion and the escape of DOX molecules from the mesochannels of AuNRs@ $\mathrm{mSiO}_{2}$ nanoparticles. ${ }^{64}$ Additionally, a rapid release of DOX was observed at $\mathrm{pH}=5.0$ with hyaluronidase, and the cumulative amount of DOX increased 

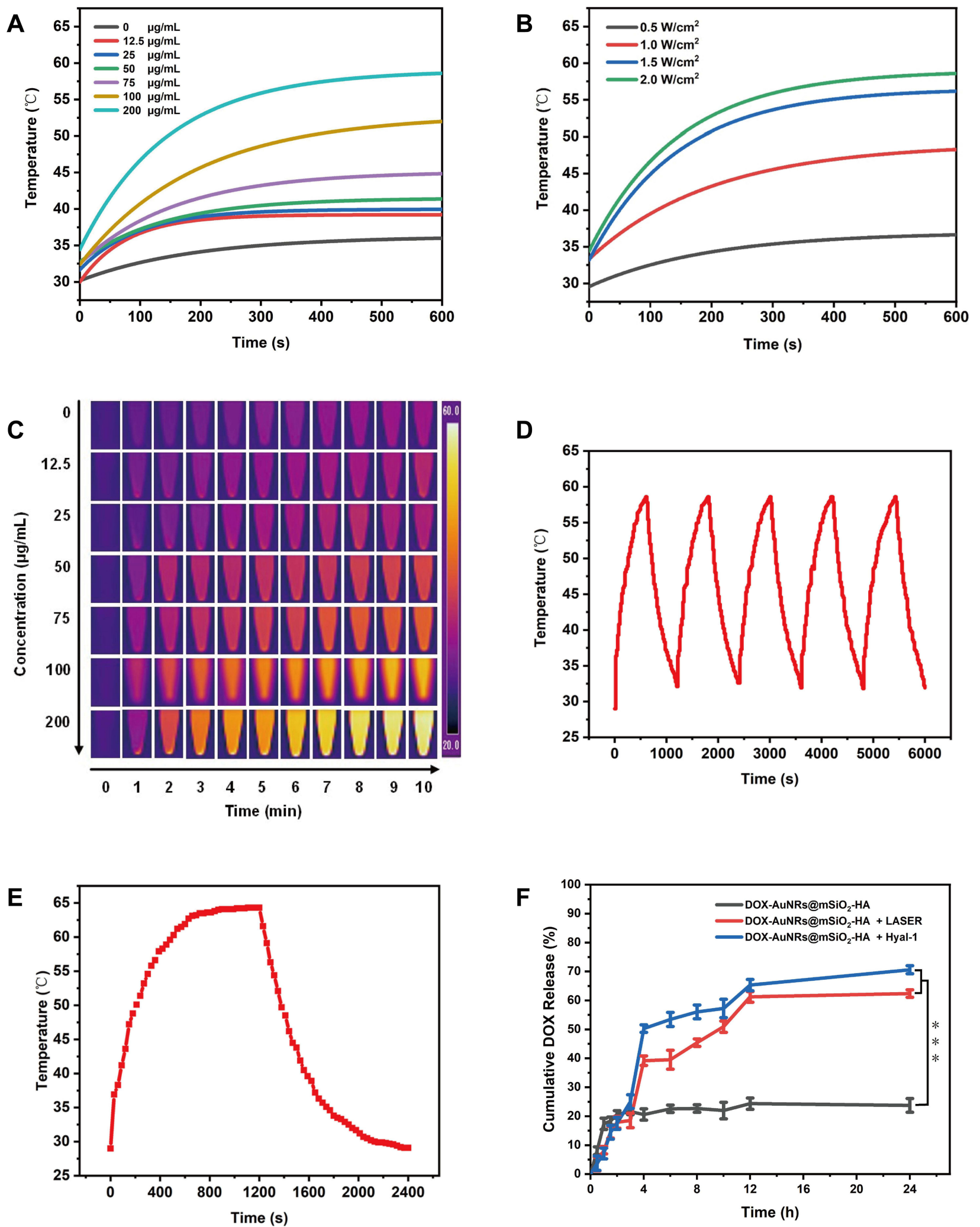

Figure 3 Temperature-rising assessment and drug release of AuNRs@mSiO ${ }_{2}$-based nanocomposites. (A) Temperature change of the AuNRs@mSiO ${ }_{2}-\mathrm{HA}$ aqueous solution under NIR laser irradiation $\left(2.0 \mathrm{~W} \mathrm{~cm}^{-2}, 808 \mathrm{~nm}\right)$. (B) Temperature elevation in the AuNRs@ $\mathrm{mSiO}_{2}-\mathrm{HA}$ aqueous solution (I.0 mL, $\left.200 \mu \mathrm{mL} \mathrm{mL}^{-1}\right)$ with NIR laser exposure as a function of power density. (C) IR thermographic maps of the cavities containing AuNRs@mSiO ${ }_{2}-\mathrm{HA}$ aqueous solution subjected to 10 min of NIR irradiation (2.0 W cm ${ }^{-2}$, $808 \mathrm{~nm}$ ). (D) Temperature evolution of AuNRs@mSiO $2-\mathrm{HA}$ aqueous solution ( $1.0 \mathrm{~mL}, 200 \mu \mathrm{g} \mathrm{mL}^{-1}$ ) over five lasers ON/OFF cycles of NIR laser irradiation (2 W cm ${ }^{-2}, 808$ $\mathrm{nm})$. (E) The photothermal response of AuNRs@mSiO $-\mathrm{HAaqueous} \mathrm{solution}\left(1.0 \mathrm{~mL}, 200 \mu \mathrm{g} \mathrm{mL} \mathrm{L}^{-1}\right.$ ) exposed by NIR laser $\left(2.0 \mathrm{~W} \mathrm{~cm}^{-2}, 808 \mathrm{~nm}\right)$ until reaching a stabilized temperature and then the laser was turned off. (F) DOX release profiles from DOX-AuNRs@mSiO $2-\mathrm{HA}$ in PBS at different $\mathrm{pH}$ values. Data are expressed as mean $\pm \mathrm{S} D(\mathrm{n}$ $=3)$. $* * * P<0.001$. 
significantly to $70.6 \%$ within $24 \mathrm{~h}$. This effect may be due to the fact that hyaluronidase has showed its capacity to degrade HA into small fragments and promote drug release. All results demonstrated that the DOX-AuNRs@mSiO $\mathrm{m}_{2}-\mathrm{HA}$ could achieve Hyal/NIR dual-responsive release, making it possible for minimizing the drug stimulation of normal cells and improving anti-tumor efficacy.

\section{In vitro PAI of AuNRs@mSiO}

The high absorption coefficient of the AuNRs@mSiO NIR region makes them effective contrast agents for PAI. As shown in Figure 4A, the PA signal of the AuNRs@ $\mathrm{mSiO}_{2}$ solutions was enhanced with the increasing concentrations of nanoparticles, displaying a concentration dependent manner. The quantitative result exhibited a well-correlated linear relationship in Figure 4B $\left(\mathrm{R}^{2}=0.97592\right)$. These results suggest that AuNRs@ $\mathrm{mSiO}_{2}$ are excellent candidates as positive PAI contrast agents.

\section{In vitro Chemo-Photothermal Therapy}

Encouraged by the excellent photothermal effect and the exceptional DOX loading capacity of AuNRs@ $\mathrm{mSiO}_{2^{-}}$ HA, its bio-application has been investigated. The biocompatibility of AuNRs@ $\mathrm{mSiO}_{2}-\mathrm{HA}$ was initially performed on HOK cells by a standard CCK-8 assay in vitro. No distinct cytotoxicity in $\mathrm{HOK}$ was observed even at higher concentrations up to $200 \mu \mathrm{g} \mathrm{mL}^{-1}$ after $24 \mathrm{~h}$ of treatment with the AuNRs@mSiO $\mathrm{m}_{2}$-HA in our tested dose range, indicating the excellent biocompatibility of blank AuNRs@ $\mathrm{mSiO}_{2}$-HA (Figure 4C). To further confirm the synergistic therapeutic effect of DOX-AuNRs@mSiO $2^{-}$ HA, CAL-27 cells were incubated with AuNRs@mSiO $2^{-}$ $\mathrm{HA}$, free DOX and DOX-AuNRs@mSiO $\mathrm{m}_{2}-\mathrm{HA}$ at various concentrations. The cytotoxicity of the DOXAuNRs@mSiO $\mathrm{m}_{2}$-HA group was significantly lower than that of the free DOX group at an equivalent concentration of DOX, which should be attributed to the sustained release of DOX molecules. As shown in Figure 4D, the cell viability of CAL-27 cells incubated with DOXAuNRs@mSiO $\mathrm{m}_{2}$-HA without NIR laser irradiation was maintained beyond $72.76 \%$ over this wide range of concentrations, further demonstrating their intrinsic biocompatibility. Such low cytotoxicity of DOX-AuNRs@mSiO ${ }_{2-}$ HA pushed us to evaluate their potential therapeutic effects on tumor cells through the desired stimulus. However, the cell viability dramatically decreased with the increased concentration of materials because of the combined effect of induced hyperthermia and released anticancer drug DOX from the liquid PCM. In contrast, the cell viability of DOX-AuNRs@mSiO $\mathrm{m}_{2}$-HA + LASER group reduced significantly. More than $90 \%$ of cancer cells were killed at a particle concentration of $25 \mu \mathrm{g} \mathrm{mL}{ }^{-1}$, when the particle concentration increased to $50 \mu \mathrm{g} \mathrm{mL}^{-1}$, almost all the CAL-27 cells were destroyed. To sum up, DOX-AuNRs@mSiO ${ }_{2}$-HA +LASER irradiation had an exceptional synergistic therapy effect. To further investigate the photothermal therapy efficiency of DOXAuNRs@mSiO ${ }_{2}-\mathrm{HA}$ in vitro, the CAL-27 cells cultured in DOX-AuNRs@ $\mathrm{mSiO}_{2}$-HA at different times were irradiated with a laser at $808 \mathrm{~nm}\left(1 \mathrm{~W} \mathrm{~cm}^{-2}\right)$. The cells were co-stained with calcein AM and propidium iodide and taken a photo by fluorescence microscopy. As shown in Figure 4E, the results of confocal fluorescence images indicate that the lethal effect of phototherapy was maintained in agreement with the CCK- 8 results.

\section{Cellular Uptake Ability}

To verify that AuNRs@mSiO 2 -HA nanoparticles could be phagocytosed, cellular uptake experiments were performed by HOK and CAL-27 cells incubated with gradient concentration of DOX-AuNRs@ $\mathrm{mSiO}_{2}$-HA for different periods of time $(0,2,4$, and $8 \mathrm{~h})$. The fluorescence intensity and content of intracellular DOX were monitored through flow cytometry. As shown in Figure $5 \mathrm{~A}-\mathrm{D}$, the DOX fluorescence intensity greatly increased with the prolonged co-incubation time or the increased concentration of materials, demonstrating that the cellular uptake was highly controllable by adjusting these parameters. These results indicate that CAL-27 cells have the capability to take DOX-AuNRs@mSiO $\mathrm{m}_{2}-\mathrm{HA}$. As the primary HA receptor, CD44 is a cell surface glycoprotein that has been implicated in various cancers and has participated in the extravasation and migration of tumor cells. The CAL-27 cells express high levels of CD44, so that DOXAuNRs@mSiO ${ }_{2}$-HA has accumulated in HA mediates tumor cell uptake.

To further examine the time-dependent cell uptake and the intracellular release behavior of DOX-loaded AuNRs@mSiO ${ }_{2}-\mathrm{HA}$, the CLSM images of CAL-27 cells incubated with DOX-loaded AuNRs@ $\mathrm{mSiO}_{2}$-HA at different time points $(2,4$ and $8 \mathrm{~h}$ ) were collected. As shown in Figure 5E, the CLSM images were obtained for the red fluorescence of DOX and the blue fluorescence of DAPI after the nuclei were stained. With the increase of the incubation time, the DOX molecules gathered in the cell nucleus gradually increased, which suggested that DOX- 
A

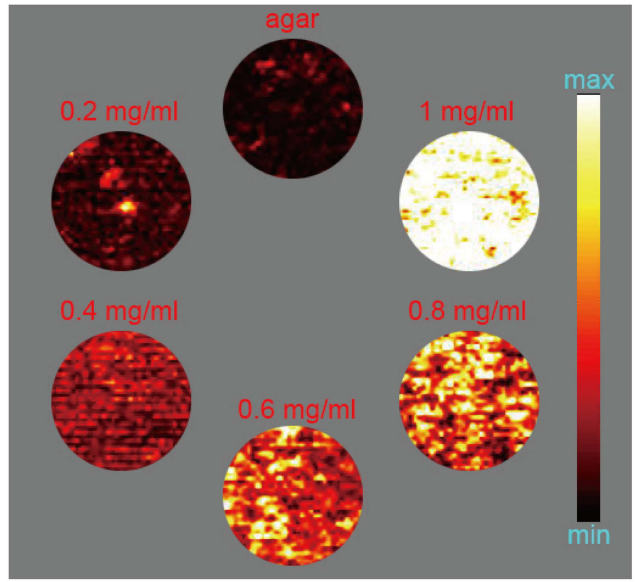

C

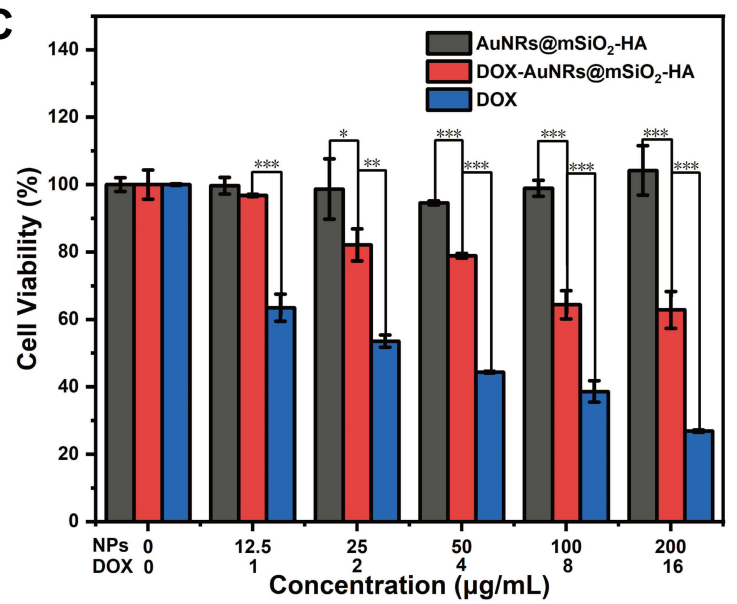

E
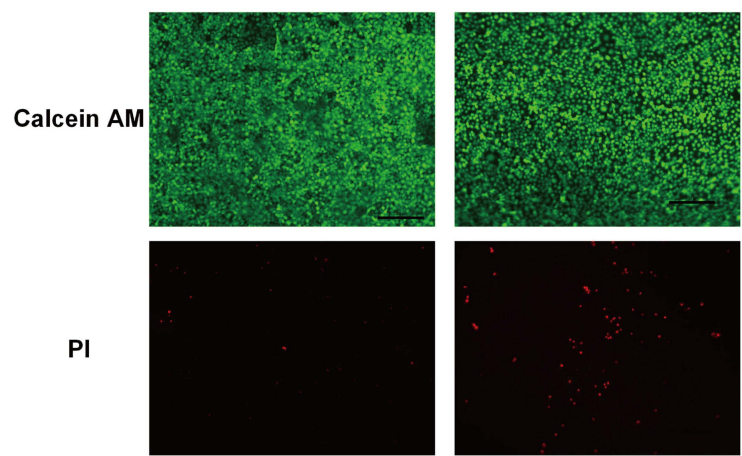

$\mathbf{P}$
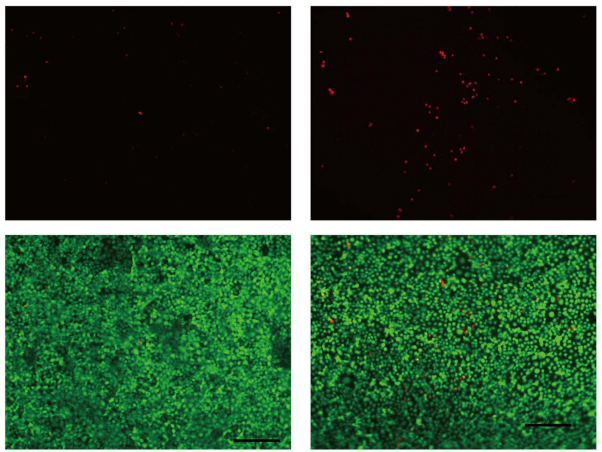

Control

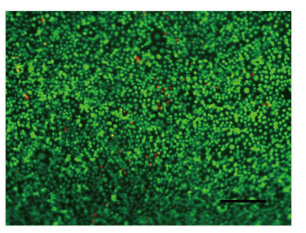

PBS + LASER
B

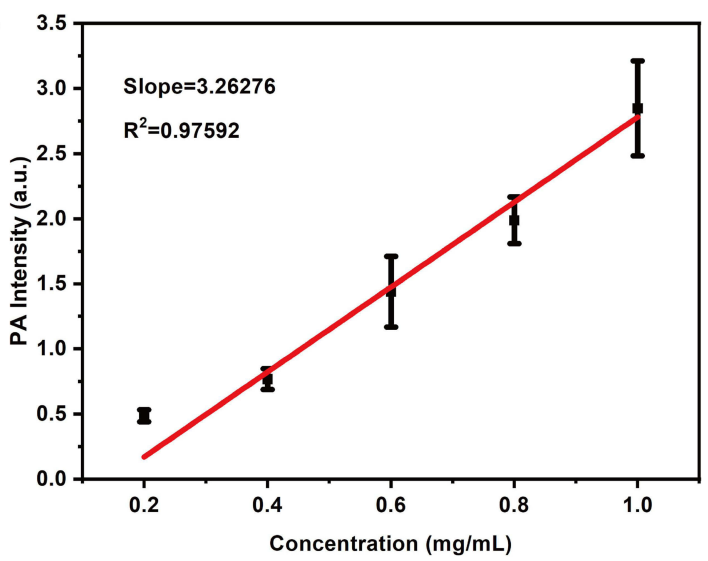

D
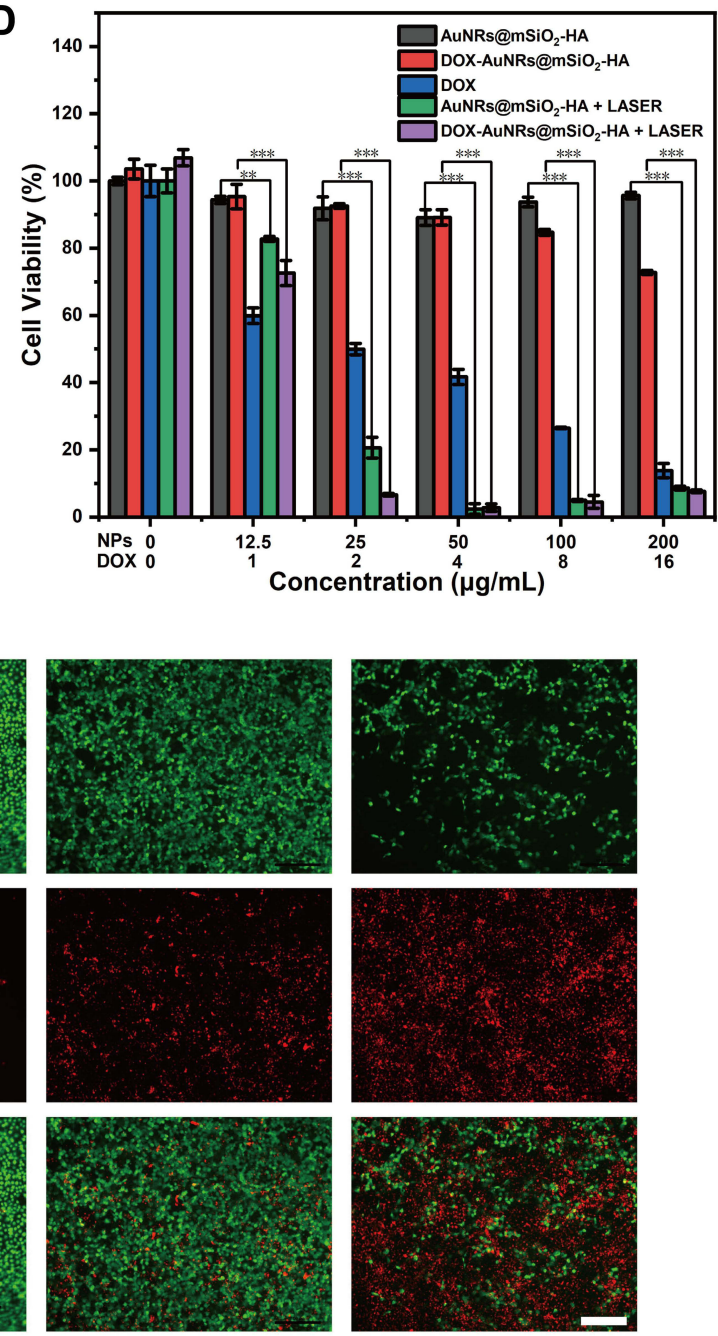

DOX-AuNRs@mSiO2-

HA

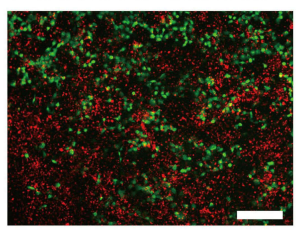

DOX-AuNRs@mSiO2HA+LASER

Figure 4 PAl and Combined therapy in vitro. (A) PA images with different AuNRs@mSiO ${ }_{2}$ concentrations. (B) PA linear relationship of PA signal intensities with different AuNRs@mSiO ${ }_{2}$ concentrations. (C) Relative cell viability of HOK cells after incubation with different concentrations of DOX, AuNRs@mSiO 2 and DOX-AuNRs@mSiO ${ }_{2}-$ $\mathrm{HA}$ for 24 h. *P< 0.05 ; **P< 0.01 ; ***P < 0.00I. (D) Relative cell viability of CAL-27 cells after incubation with different concentrations of DOX, AuNRs@mSiO 2 and DOXAuNRs@mSiO 2 - $\mathrm{HA}$ for $24 \mathrm{~h}$, followed by irradiation with $808 \mathrm{~nm}$ laser $\left(\mathrm{IW} \mathrm{cm}{ }^{-2}\right)$ for $5 \mathrm{~min}$. **P < $0.0 \mathrm{l}$; ***P<0.00I. (E) Fluorescence microscope images of Calcein AM (green fluorescence, live cells) and Propidium iodide (PI, red fluorescence, dead cells) costained CAL-27 cells incubated with PBS, LASER, DOX-AuNRs@mSiO 2 -HA and DOX-AuNRs@mSiO 2 -HA + LASER $\left(100 \mu \mathrm{gL}^{-1}\right)$. The scale bar is $100 \mu \mathrm{m}$. 
A

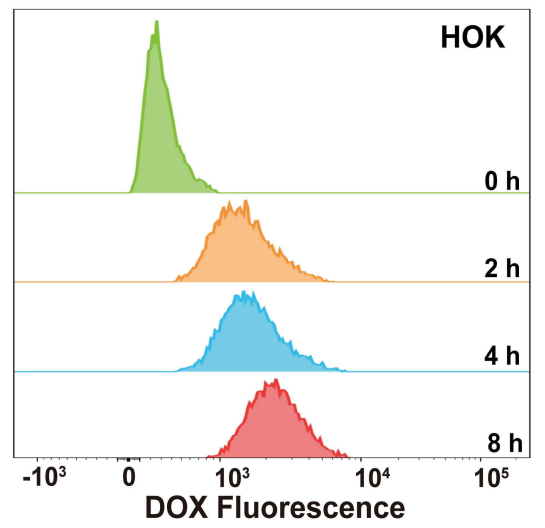

C

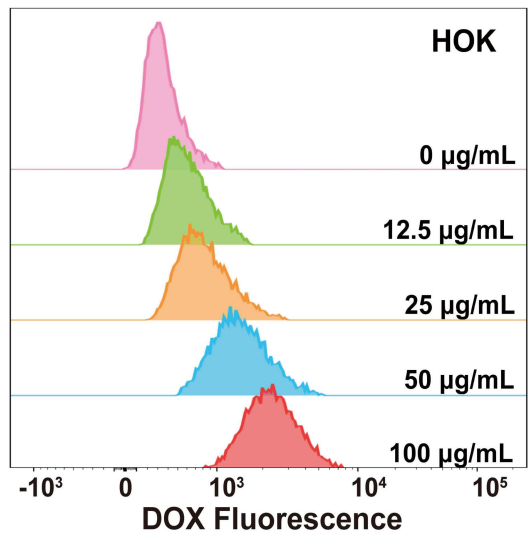

B

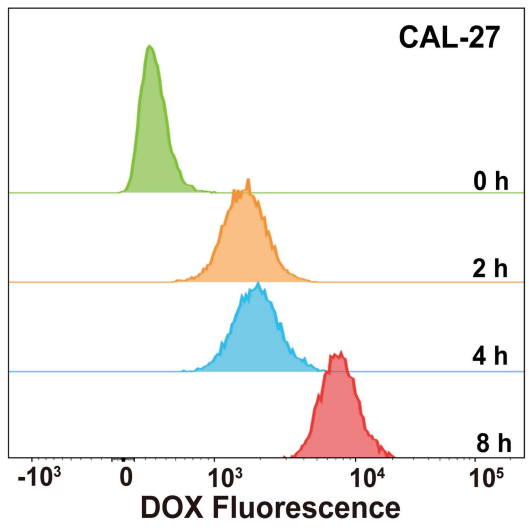

D

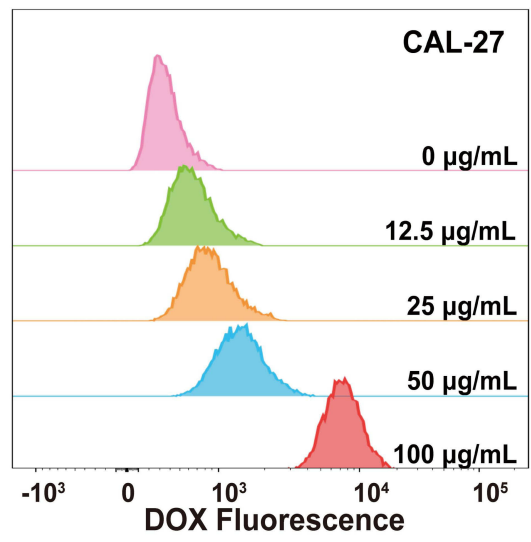

E

$2 \mathrm{~h}$
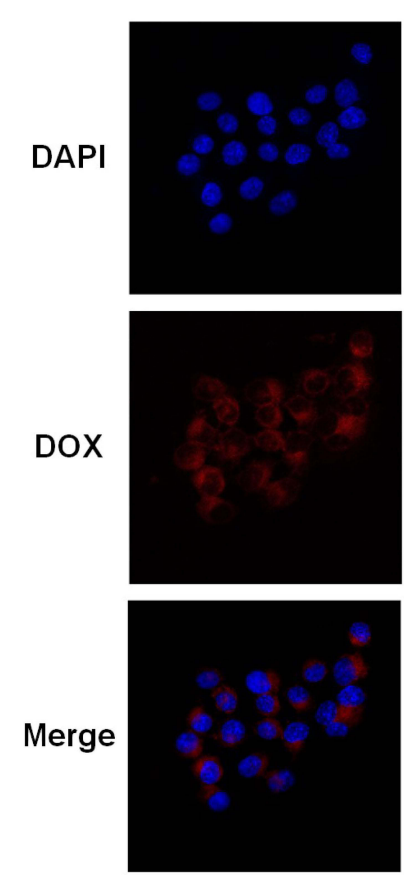

$4 \mathrm{~h}$
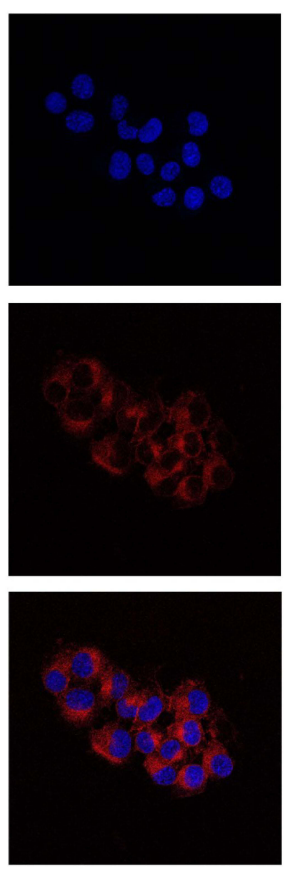

$8 \mathrm{~h}$
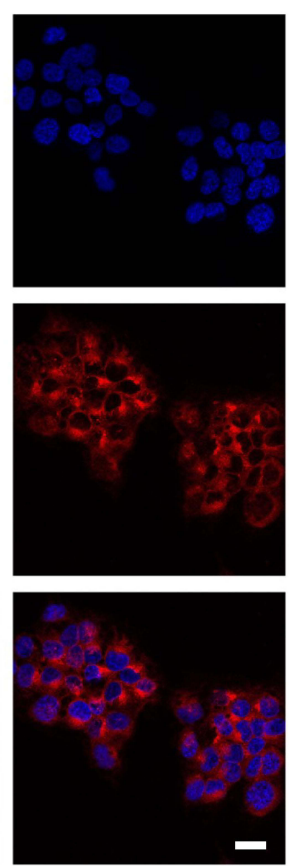

Figure 5 Cellular uptake study. (A) Intracellular DOX fluorescence intensity of HOK cells measured by flow cytometry after incubating cells with I00 $\mu \mathrm{g} \mathrm{mL}^{-1} \mathrm{DOX}$ AuNRs@mSiO $-\mathrm{HA}$ for 0, 2, 4 and $8 \mathrm{~h}$. (B) Intracellular DOX fluorescence intensity of CAL-27 cells measured by flow cytometry after incubating cells with $100 \mu \mathrm{g} \mathrm{mL}^{-1}$ DOX-AuNRs@mSiO $-\mathrm{HA}$ for 0, 2, 4 and $8 \mathrm{~h}$. (C) Intracellular DOX fluorescence intensity of HOK cells measured by flow cytometry after incubating cells with DOXAuNRs@mSiO $2-\mathrm{HA}$ for $8 \mathrm{~h}$. (D) Intracellular DOX fluorescence intensity of CAL-27 cells measured by flow cytometry after incubating cells with DOX-AuNRs@mSiO 2 -HA for 8 h. (E) Confocal laser scanning microscope (CLSM) images of CAL-27 cells showing colocalization of nucleus and DOX filmed at 2, 4, $8 \mathrm{~h}$ after incubating cells with DOX-AuNRs@mSiO $2-\mathrm{HA}$ at $37^{\circ} \mathrm{C}$. Nucleus stained with DAPI. The scale bar is $20 \mu \mathrm{m}$. 
loaded nanoparticles could facilitate cellular internalization and final delivery of DOX to the cell nuclei. It can be concluded that the DOX-AuNRs@mSiO 2 - $\mathrm{HA}$ has great potential as a promising nanocarrier for the administration of drugs against oral cancer cells.

\section{In vivo PAI of AuNRs@mSiO $-\mathrm{HA}$}

Take advantage of the high absorption of NIR, AuNRs@mSiO 2 -HA can also be used as a contrast agent in PAI, which provides the local distribution of nanoparticles in the tumor region. As shown in Figure 6A, CAL-27 tumor-bearing mice with a tumor volume of approximately $100 \mathrm{~mm}^{3}$ were randomly divided into four groups of five mice per group. With the guidance of PAI, AuNRs@mSiO 2 -HA suspensions (200 $\left.\mu \mathrm{L}, 1 \mathrm{mg} \mathrm{mL}{ }^{-1}\right)$ were injected into tumor-bearing mice by injection into the tail vein. PA signals in the tumor region were recorded at different time points $(0,2,4,8,12,24 \mathrm{~h})$. As shown in Figure $6 \mathrm{~B}$ and $\mathrm{C}$, the PA signal increased gradually within the first 8-h post-injection at tumor site and then decreased, which indicated that the maximum nanoparticles enrichment in the tumor region was achieved at 8 $\mathrm{h}$ post-injection.

\section{In vivo Chemo-Photothermal Therapy}

Inspired by the excellent accumulation and anti-tumor effect in vitro of DOX-AuNRs@ $\mathrm{mSiO}_{2}-\mathrm{HA}$, the efficacy of in vivo therapy was further evaluated in mice. After injection of particulate suspensions through the caudal vein, the tumors are completely exposed to $808 \mathrm{~nm}$ laser at $8 \mathrm{~h}$ after the injection. The temperature of the tumor site in the DOX-AuNRs@ $\mathrm{mSiO}_{2}$-HA group increased from 28.6 to $55.7{ }^{\circ} \mathrm{C}$ after laser irradiation (Figure 6D), while the temperature of the mice in the PBS group did not increase significantly after $5 \mathrm{~min}$ of laser irradiation. As shown in Figure 6E, the digital photographs of tumors on the 12th day after treatment show that tumors in DOXAuNRs@mSiO 2 -HA + LASER group are completely ablated. The body weight curves of the four groups showed no significant difference from each other, which implied that treatments did not affect the normal development of the mice during our experiment (Figure 6F). We found that tumor volumes in the control groups increased rapidly during the experiment (Figures 6G and 7A). In comparison, the DOX-AuNRs@mSiO 2 -HA and PBS+LASER groups showed a slower tumor growth rate than the control groups, which means the therapeutic effect of single chemotherapy or PTT alone was limited. When mice injected with DOX-
AuNRs@mSiO 2 -HA were exposed to the laser, the tumor growth was significantly suppressed within the first 12 days. Moreover, the tumors were gradually ablated in the last 8 days of the experiment, which indicated DOXAuNRs@mSiO 2 -HA + LASER had a better anti-tumor and therapeutic efficacy than any other group, and the synergistic effect of PTT and chemotherapy is effective. These results agree fairly well with the tumor growth curves. As illustrated in Figure 7B, the follow-up time for these treated mice was recorded. After irradiation with NIR light, the DOX-AuNRs@ $\mathrm{mSiO}_{2}$-HA group was the best in terms of the survival rate among all the experimental groups. $\mathrm{Xu}$ and co-authors found that the multifunctional nanoplatform loaded with DOX was used to treat the tumorbearing mouse model of breast cancer, in contrast, the chemo-photothermal therapy group showed the best antitumor efficacy. ${ }^{65}$ Furthermore, H\&E staining of tumor slices showed that most of the cells in the tumors of mice of DOX-AuNRs@mSiO $\mathrm{m}_{2}$-HA +LASER group were severely damaged, while the cells in the tumors of mice of the other treatment groups were only partially destroyed (Figures 7C and S8). The above results indicated that DOXAuNRs@mSiO ${ }_{2}$-HA had good photothermal properties and could carry out effective Chemo-Photothermal therapy. To further confirm the biocompatibility of DOXAuNRs@mSiO 2 -HA, mice were dissected within 30 days of treatment, and their tumors and major organs were subjected to histological analysis. As shown in Figure 7D, there was no obvious organ damage and inflammation compared to the PBS group. The safety and low toxicity of DOXAuNRs@mSiO $\mathrm{m}_{2}$-HA were also demonstrated to be good. These results suggested that DOX-AuNRs@mSiO $\mathrm{miO}_{2}-\mathrm{HA}$ nanoparticles could serve as an effective platform for cancer chemo-photothermal therapy in vivo relatively low systemic toxicity.

\section{Conclusion}

In conclusion, we have successfully fabricated novel DOXAuNRs@mSiO $\mathrm{m}_{2}$-HA which possesses ultrahigh strong PAI signal, prominent photothermal conversion and drug loading ability. The in vitro cellular and in vivo animal experiments demonstrated that DOX-AuNRs@mSiO $2-\mathrm{HA}$ could simultaneously exert chemotherapy and photothermal effect under the laser irradiation. Overall, this AuNRs@mSiO ${ }_{2}$-HA PTT platform combined with chemotherapy showed massive potential as a nanotheranostic for the diagnosis and treatment of precision cancer therapy. However, the animal model of our study is the model of 

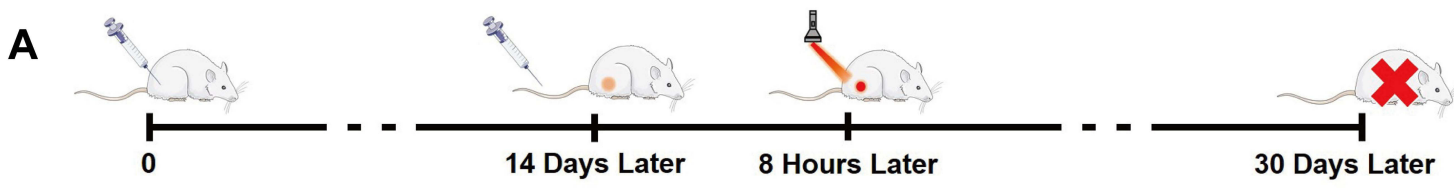

B
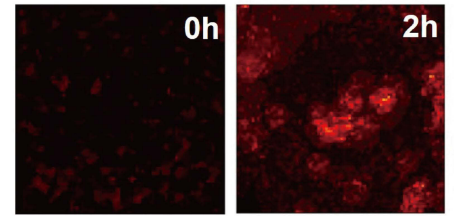

8h
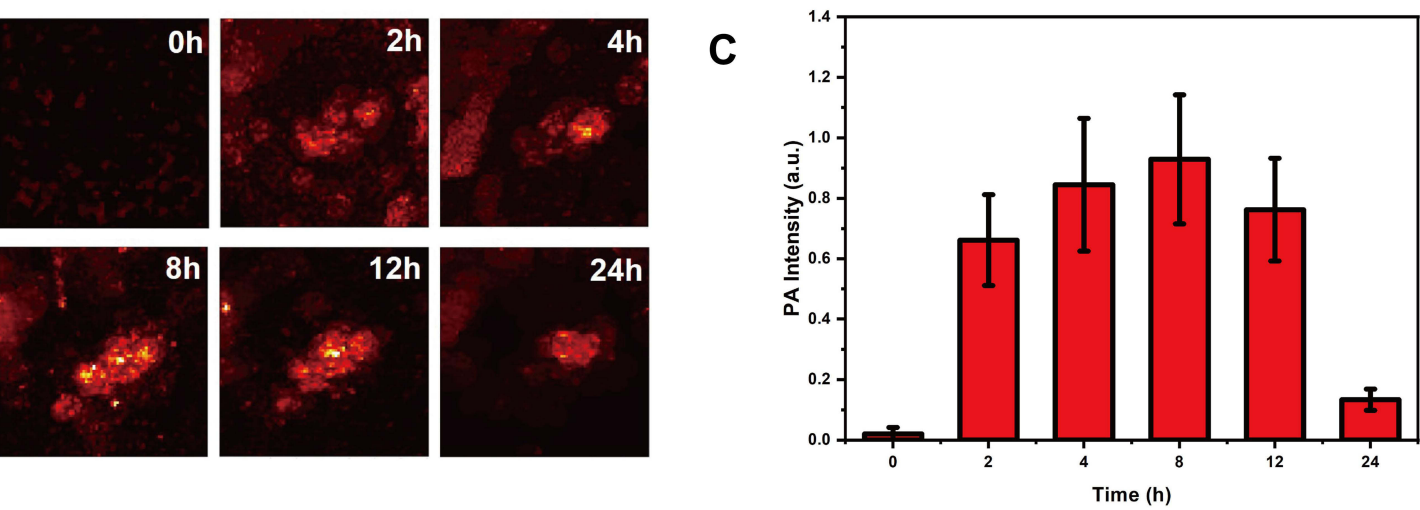

D

PBS + LASER

DOX-AuNRs@mSiOz -HA +LASER
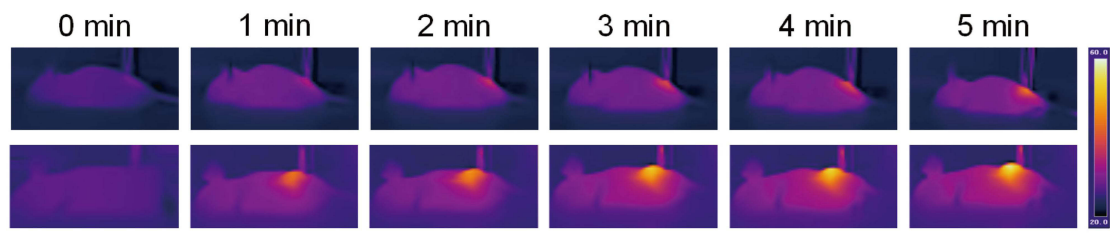

E

DOX-AuNRs@mSiO2-HA +LASER

DOX-AuNRs@mSiO2-HA

PBS + LASER

Control

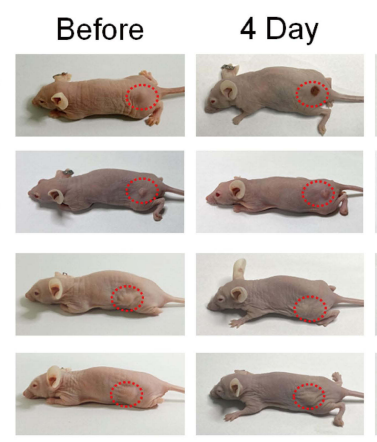

$\mathbf{F}$

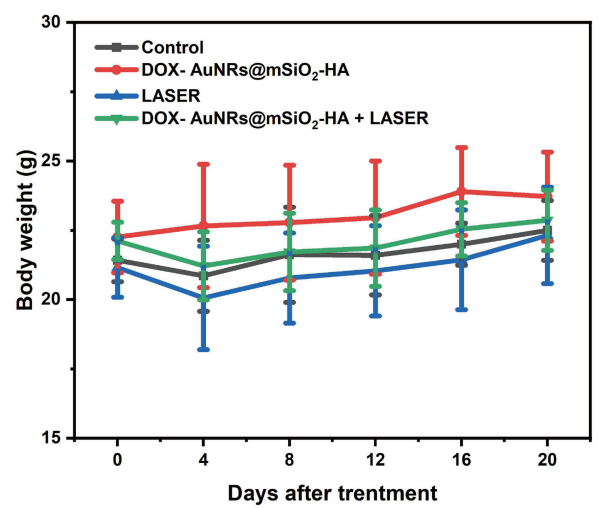

8 Day

12 Day

16 Day

20 Day
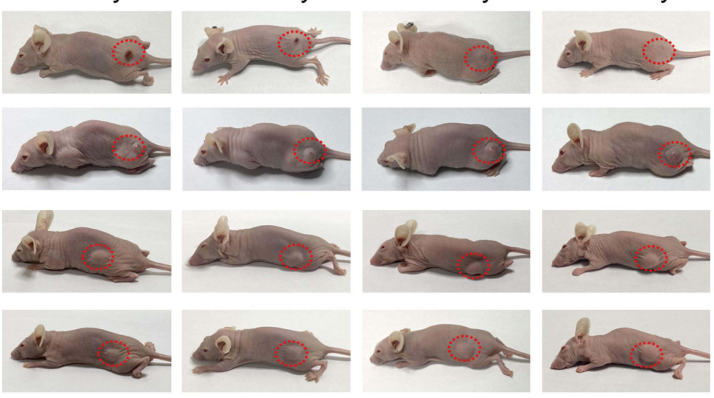

G

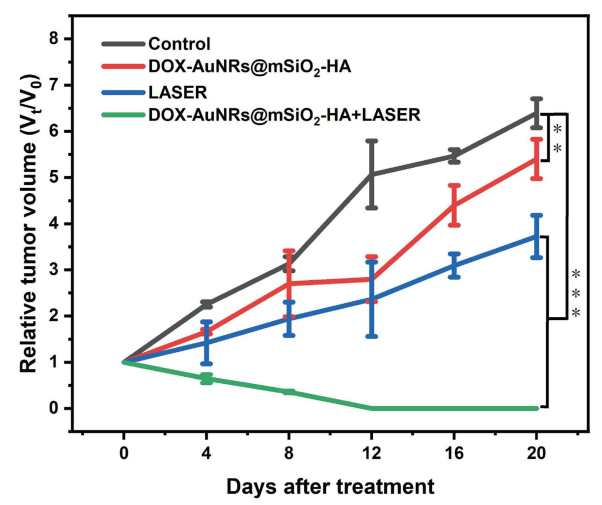

Figure 6 In vivo PAl and PTT of CAL-27 tumor-bearing mice. (A) Scheme of CAL-27 tumor-bearing mouse development and Chemo-Photothermal Therapy. (B) PAl in the tumor region after intravenously injected DOX-AuNRs@ $\mathrm{mSiO}_{2}-\mathrm{HA}$ are recorded at different time points after intravenous administration. (C) PA intensity in the tumor region after intravenously injected DOX-AuNRs@mSiO 2 - $\mathrm{HA}$ are recorded at different time points after intravenous administration. (D) Photothermal images of CAL-27 tumor-bearing mice injected with PBS and DOX-AuNRs@mSiO ${ }_{2}-\mathrm{HA}\left(200 \mu \mathrm{L}, 1 \mathrm{mg} \mathrm{mL}{ }^{-1}\right)$ under NIR laser irradiation $\left(808 \mathrm{~nm}, 2 \mathrm{~W} \mathrm{~cm}{ }^{-2}\right)$ for 5 min at 6-h post-injection. (E) Representative photos of CAL-27 tumor-bearing mice after different days before $(0 \mathrm{~d})$ and after $(4,8,12,16$ and $20 \mathrm{~d})$ different treatments. (F) Average body weights of mice from each group after various treatments: (I) Control, without treatment; (2) DOX-AuNRs@mSiO $2-H A$, injection of DOX-AuNRs@mSiO ${ }_{2}-\mathrm{HA}$ only; (3) LASER, irradiation with laser only; (4) DOX-AuNRs@mSiO $2-\mathrm{HA}+\mathrm{LASER}$, injection of DOXAuNRs@ $\mathrm{mSiO}_{2}-\mathrm{HA}$ and irradiation with a laser. $(\mathbf{G})$ Relative volumes of tumors after the above treatments, normalized to their initial volumes. $* * P<0.0 \mathrm{I}$; $* * * P<0.00 \mathrm{I}$. 
A

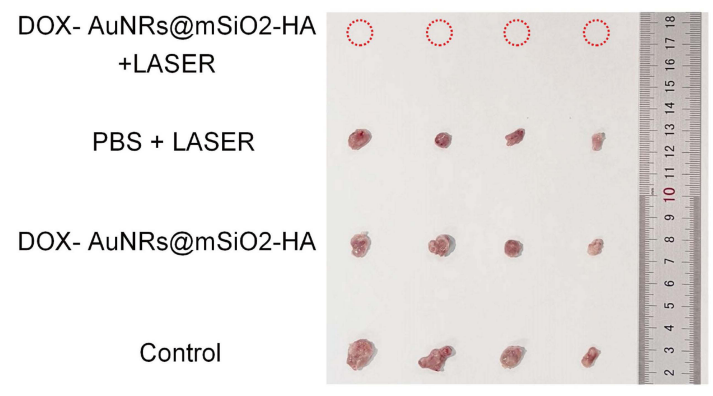

B

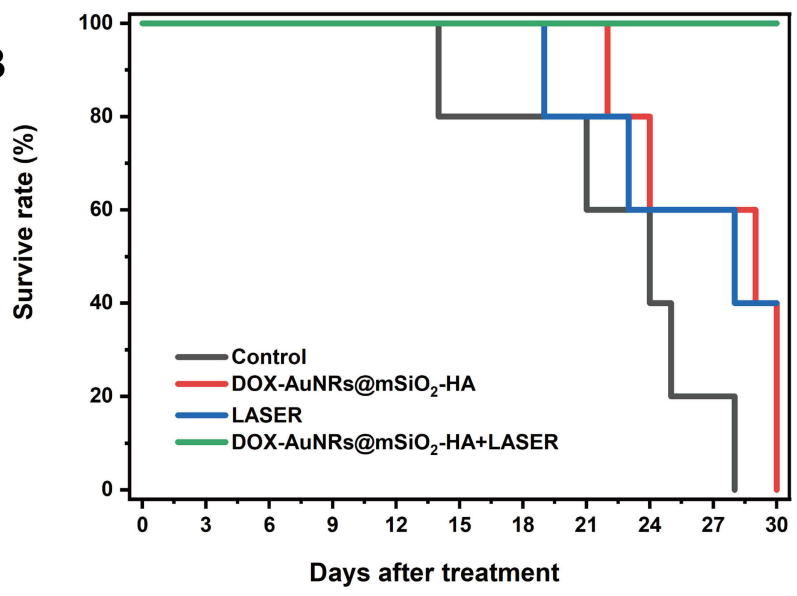

C

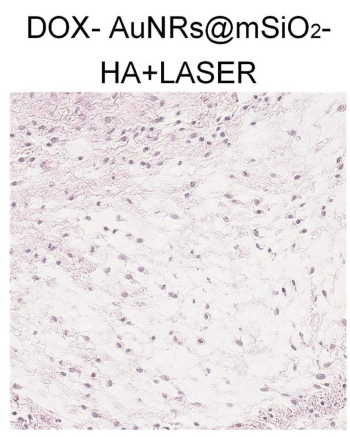

PBS + LASER

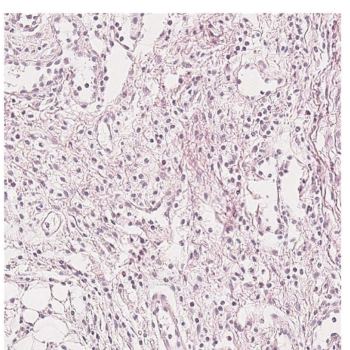

DOX-AuNRs@mSiO2-HA

Control
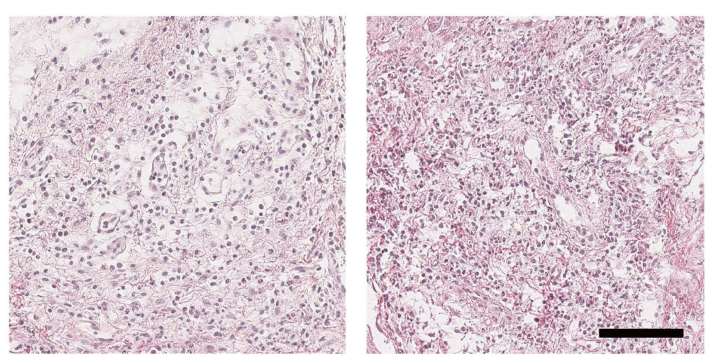

D

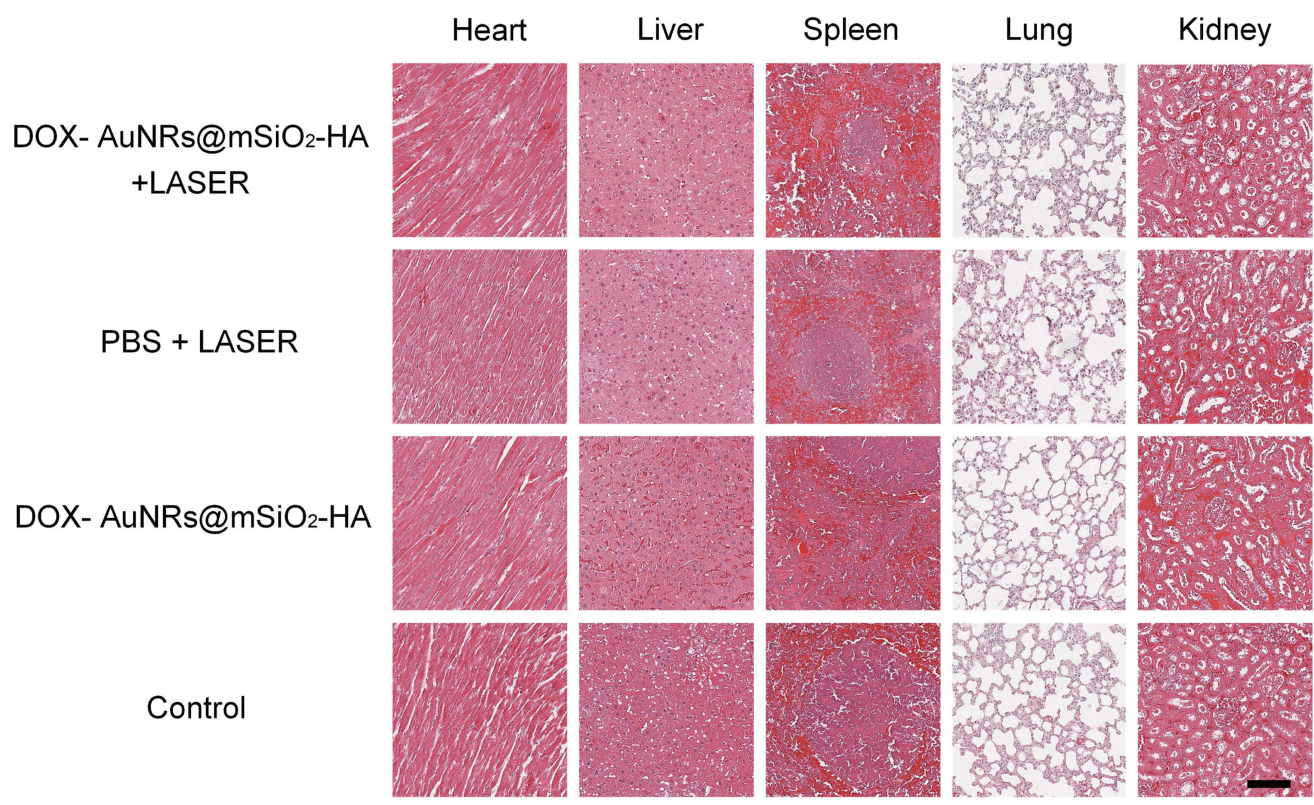

Figure 7 In vivo antitumor activity of different types of CAL-27 tumor-bearing mice. (A) Optical images of the dissected tumors from CAL-27 tumor-bearing mice in different treatment groups at day 30 after sacrificing the mice. (B) Survival curves of CAL-27 tumor-bearing mice in various groups after NIR-light irradiation treatment. (C) Histological section of tumor stained with hematoxylin and eosin after intravenous administration of DOX-AuNRs@mSiO 2 -HA. The scale bar is I00 $\mu$ m. (D) Histological section of vital organs (heart, liver, spleen, lung and kidney) stained with hematoxylin and eosin after intravenous administration of DOX-AuNRs@mSiO ${ }_{2}-\mathrm{HA}_{\mathrm{A}}$. The scale bar is $100 \mu \mathrm{m}$.

back ectopic transplanted tumor in tumor-bearing mice, which is mainly limited by the imperfection of the imaging device and treatment device. Moving forward, we will further enhance experimental equipment and conduct comprehensive research on the diagnosis and treatment of primary oral cancer. 


\section{Acknowledgments}

This research is supported by the Guangdong Basic and Applied Basic Research Foundation (2020A1515011104), the Project of Guangdong Science and Technology Plan (2020A1414010329), the Guangzhou Science and Technology Plan Project (202102020140), the project of Guangzhou Liwan Science and Technology Plan (201904005), and the National Natural Science Foundation of China (11704082).

\section{Disclosure}

The authors reported no conflicts of interest in this work.

\section{References}

1. Carlisle JW, Ramalingam SS. A banner year for immunotherapy and targeted therapy. Nat Rev Clin Oncol. 2019;16(2):79-80. doi:10.1038/s41571-018-0138-4

2. Li C, Zhang Y, Li Z, et al. Light-responsive biodegradable nanorattles for cancer theranostics. Adv Mater. 2018;30(8). doi:10.1002/ adma. 201706150

3. Li Y, Dang J, Liang Q, Yin L. Thermal-responsive carbon monoxide (CO) delivery expedites metabolic exhaustion of cancer cells toward reversal of chemotherapy resistance. ACS Cent Sci. 2019;5 (6):1044-1058. doi:10.1021/acscentsci.9b00216

4. Rothenberg SM, Ellisen LW. The molecular pathogenesis of head and neck squamous cell carcinoma. $J$ Clin Invest. 2012;122 (6):1951-1957. doi:10.1172/jci59889

5. Chi AC, Day TA, Neville BW. Oral cavity and oropharyngeal squamous cell carcinoma-an update. CA Cancer J Clin. 2015;65 (5):401-421. doi:10.3322/caac. 21293

6. Grafton-Clarke C, Chen KW, Wilcock J. Diagnosis and referral delays in primary care for oral squamous cell cancer: a systematic review. $\mathrm{Br}$ J Gen Pract. 2019;69(679):e112-e126. doi:10.3399/bjgp18X700205

7. Lousada-Fernandez F, Rapado-Gonzalez O, Lopez-Cedrun JL, LopezLopez R, Muinelo-Romay L, Suarez-Cunqueiro MM. Liquid biopsy in oral cancer. Int J Mol Sci. 2018;19(6):1704. doi:10.3390/ijms19061704

8. Ghosh RD, Pattatheyil A, Roychoudhury S. Functional landscape of dysregulated MicroRNAs in oral squamous cell carcinoma: clinical implications. Front Oncol. 2020;10:619. doi:10.3389/ fonc.2020.00619

9. Kosugi A, Kasahara M, Yang L, Nakamura-Takahashi A, Shibahara T, Mori T. Method for diagnosing neoplastic lesions by quantitative fluorescence value. Sci Rep. 2019;9(1):7833. doi:10.1038/s41598-019-44287-z

10. Wezgowiec J, Wieczynska A, Wieckiewicz W, et al. Polish propolis-chemical composition and biological effects in tongue cancer cells and macrophages. Molecules. 2020;25(10):2426. doi: $10.3390 /$ molecules 25102426

11. Bray F, Ferlay J, Soerjomataram I, Siegel RL, Torre LA, Jemal A. Global cancer statistics 2018: GLOBOCAN estimates of incidence and mortality worldwide for 36 cancers in 185 countries. CA Cancer J Clin. 2018;68(6):394-424. doi:10.3322/caac.21492

12. Li C, Liu S, Yan R, Han N, Wong KK, Li L. CD54-NOTCH1 axis controls tumor initiation and cancer stem cell functions in human prostate cancer. Theranostics. 2017;7(1):67-80. doi:10.7150/ thno. 16752

13. Grohl J, Schellenberg M, Dreher K, Maier-Hein L. Deep learning for biomedical photoacoustic imaging: a review. Photoacoustics. 2021;22:100241. doi:10.1016/j.pacs.2021.100241
14. Beard P. Biomedical photoacoustic imaging. Interface Focus. 2011;1 (4):602-631. doi:10.1098/rsfs.2011.0028

15. Hoelen CG, de Mul FF, Pongers R, Dekker A. Three-dimensional photoacoustic imaging of blood vessels in tissue. Opt Lett. 1998;23 (8):648-650. doi:10.1364/ol.23.000648

16. Xiong J, Feng J, Qiu L, et al. SDF-1-loaded PLGA nanoparticles for the targeted photoacoustic imaging and photothermal therapy of metastatic lymph nodes in tongue squamous cell carcinoma. Int J Pharm. 2019;554:93-104. doi:10.1016/j.ijpharm.2018.10.064

17. Fatakdawala H, Poti S, Zhou F, et al. Multimodal in vivo imaging of oral cancer using fluorescence lifetime, photoacoustic and ultrasound techniques. Biomed Opt Express. 2013;4(9):1724-1741. doi:10.1364/ BOE.4.001724

18. Muhanna N, Jin CS, Huynh E, et al. Phototheranostic porphyrin nanoparticles enable visualization and targeted treatment of head and neck cancer in clinically relevant models. Theranostics. 2015;5 (12):1428-1443. doi:10.7150/thno.13451

19. Tabish TA, Dey P, Mosca S, et al. Smart gold nanostructures for light mediated cancer theranostics: combining optical diagnostics with photothermal therapy. Adv Sci. 2020;7(15):1903441. doi:10.1002/ advs.201903441

20. Sun J, Li Y, Teng Y, Wang S, Guo J, Wang C. NIR-controlled HSP90 inhibitor release from hollow mesoporous nanocarbon for synergistic tumor photothermal therapy guided by photoacoustic imaging. Nanoscale. 2020;12(27):14775-14787. doi:10.1039/d0nr02896g

21. Cai K, Zhang W, Zhang J, Li H, Han H, Zhai T. Design of gold hollow nanorods with controllable aspect ratio for multimodal imaging and combined chemo-photothermal therapy in the second near-infrared window. ACS Appl Mater Interfaces. 2018;10 (43):36703-36710. doi:10.1021/acsami.8b12758

22. He Y, Wan J, Yang Y, et al. Multifunctional polypyrrole-coated mesoporous $\mathrm{TiO} 2$ nanocomposites for photothermal, sonodynamic, and chemotherapeutic treatments and dual-modal ultrasound/photoacoustic imaging of tumors. Adv Healthc Mater. 2019;8(9):e1801254. doi:10.1002/adhm.201801254

23. Hirsch LR, Stafford RJ, Bankson JA, et al. Nanoshell-mediated near-infrared thermal therapy of tumors under magnetic resonance guidance. Proc Natl Acad Sci U S A. 2003;100(23):13549-13554. doi:10.1073/pnas.2232479100

24. Chu KF, Dupuy DE. Thermal ablation of tumours: biological mechanisms and advances in therapy. Nat Rev Cancer. 2014;14 (3):199-208. doi:10.1038/nrc3672

25. Nam J, Son S, Ochyl LJ, Kuai R, Schwendeman A, Moon JJ. Chemophotothermal therapy combination elicits anti-tumor immunity against advanced metastatic cancer. Nat Commun. 2018;9(1):1074. doi:10.1038/s41467-018-03473-9

26. Poustchi F, Amani H, Ahmadian Z, et al. Combination therapy of killing diseases by injectable hydrogels: from concept to medical applications. Adv Healthc Mater. 2021;10(3):e2001571. doi:10.1002/adhm.202001571

27. Srinivas PR, Barker P, Srivastava S. Nanotechnology in early detection of cancer. Lab Invest. 2002;82(5):657-662. doi:10.1038/ labinvest. 3780460

28. Forrest ML, Won CY, Malick AW, Kwon GS. In vitro release of the mTOR inhibitor rapamycin from poly (ethylene glycol)-b-poly (epsilon-caprolactone) micelles. $J$ Control Release. 2006;110 (2):370-377. doi:10.1016/j.jconrel.2005.10.008

29. Wang M, Thanou M. Targeting nanoparticles to cancer. Pharmacol Res. 2010;62(2):90-99. doi:10.1016/j.phrs.2010.03.005

30. Yingchoncharoen P, Kalinowski DS, Richardson DR. Lipid-based drug delivery systems in cancer therapy: what is available and what is yet to come. Pharmacol Rev. 2016;68(3):701-787. doi:10.1124/ pr.115.012070

31. Qiu L, Larson TA, Vitkin E, et al. Gold nanorod light scattering labels for biomedical imaging. Biomed Opt Express. 2010;1 (1):135-142. doi:10.1364/BOE.1.000135 
32. Jabeen F, Najam-ul-Haq M, Javeed R, Huck CW, Bonn GK. Aunanomaterials as a superior choice for near-infrared photothermal therapy. Molecules. 2014;19(12):20580-20593. doi:10.3390/ molecules 191220580

33. Begines B, Alcudia A, Aguilera-Velazquez R, et al. Design of highly stabilized nanocomposite inks based on biodegradable polymer-matrix and gold nanoparticles for Inkjet Printing. Sci Rep. 2019;9(1):16097. doi:10.1038/s41598-019-52314-2

34. Begines B, Alcudia A, Aguilera-Velazquez R, et al. Author correction: design of highly stabilized nanocomposite inks based on biodegradable polymer-matrix and gold nanoparticles for Inkjet Printing. Sci Rep. 2020;10(1):19793. doi:10.1038/s41598-020-76435-1

35. Liu L, Xie HJ, Mu LM, et al. Functional chlorin gold nanorods enable to treat breast cancer by photothermal/photodynamic therapy Int J Nanomedicine. 2018;13:8119-8135. doi:10.2147/IJN.S186974

36. Yang Z, Song J, Dai Y, et al. Self-assembly of semiconducting-plasmonic gold nanoparticles with enhanced optical property for photoacoustic imaging and photothermal therapy. Theranostics. 2017;7(8):2177-2185. doi:10.7150/thno.20545

37. Zhang K, Zhou D, Wang Z, Zhang Y, He P. Hybrid mesoporous silica nanospheres modified by poly (NIPAM-co-AA) for drug delivery. Nanotechnology. 2019;30(35):355604. doi:10.1088/1361-6528/ ab209d

38. Kumar B, Murali A, Bharath AB, Giri S. Guar gum modified upconversion nanocomposites for colorectal cancer treatment through enzyme-responsive drug release and NIR-triggered photodynamic therapy. Nanotechnology. 2019;30(31):315102. doi:10.1088/1361$6528 / \mathrm{ab} 116 \mathrm{e}$

39. Li Z, Zhang Y, Feng N. Mesoporous silica nanoparticles: synthesis, classification, drug loading, pharmacokinetics, biocompatibility, and application in drug delivery. Expert Opin Drug Deliv. 2019;16 (3):219-237. doi:10.1080/17425247.2019.1575806

40. Ramasamy T, Ruttala HB, Sundaramoorthy P, et al. Multimodal selenium nanoshell-capped $\mathrm{Au} @ \mathrm{mSiO} 2$ nanoplatform for NIR-responsive chemo-photothermal therapy against metastatic breast cancer. NPG Asia Mater. 2018;10(4):197-216. doi:10.1038/ s41427-018-0034-5

41. Tarn D, Ashley CE, Xue M, Carnes EC, Zink JI, Brinker CJ. Mesoporous silica nanoparticle nanocarriers: biofunctionality and biocompatibility. Acc Chem Res. 2013;46(3):792-801. doi:10.1021/ ar3000986

42. Lee JE, Lee N, Kim H, et al. Uniform mesoporous dye-doped silica nanoparticles decorated with multiple magnetite nanocrystals for simultaneous enhanced magnetic resonance imaging, fluorescence imaging, and drug delivery. J Am Chem Soc. 2010;132(2):552-557. doi: $10.1021 / \mathrm{ja} 905793 \mathrm{q}$

43. Fang Z, Li X, Xu Z, et al. Hyaluronic acid-modified mesoporous silica-coated superparamagnetic $\mathrm{Fe} 3 \mathrm{O} 4$ nanoparticles for targeted drug delivery. Int $J$ Nanomedicine. 2019;14:5785-5797. doi:10.2147/IJN.S213974

44. Fong YT, Chen CH, Chen JP. Intratumoral delivery of doxorubicin on folate-conjugated graphene oxide by in-situ forming thermo-sensitive hydrogel for breast cancer therapy. Nanomaterials. 2017;7(11). doi: 10.3390/nano 7110388

45. Chen J, Zhou J, Lu J, Xiong H, Shi X, Gong L. Significance of CD44 expression in head and neck cancer: a systemic review and meta-analysis. BMC Cancer. 2014;14:15. doi:10.1186/1471-240714-15

46. Wang Z, Sau S, Alsaab HO, Iyer AK. CD44 directed nanomicellar payload delivery platform for selective anticancer effect and tumor specific imaging of triple negative breast cancer. Nanomedicine. 2018;14(4):1441-1454. doi:10.1016/j.nano.2018.04.004

47. Huang S, Li C, Wang W, et al. A54 peptide-mediated functionalized gold nanocages for targeted delivery of DOX as a combinational photothermal-chemotherapy for liver cancer. Int $J$ Nanomedicine. 2017;12:5163-5176. doi:10.2147/IJN.S131089
48. Ramasamy T, Ruttala HB, Gupta B, et al. Smart chemistry-based nanosized drug delivery systems for systemic applications: a comprehensive review. J Control Release. 2017;258:226-253. doi:10.1016/j.jconrel.2017.04.043

49. Holub AR, Huo A, Patel K, Thakore V, Chhibber P, Erogbogbo F. Assessing advantages and drawbacks of rapidly generated ultra-large 3D breast cancer spheroids: studies with chemotherapeutics and nanoparticles. Int $J$ Mol Sci. 2020;21(12):4413. doi:10.3390/ ijms 21124413

50. Singh N, Sallem F, Mirjolet C, et al. Polydopamine modified superparamagnetic iron oxide nanoparticles as multifunctional nanocarrier for targeted prostate cancer treatment. Nanomaterials. 2019;9(2):138. doi:10.3390/nano9020138

51. Li L, He S, Yu L, et al. Codelivery of DOX and siRNA by folate-biotin-quaternized starch nanoparticles for promoting synergistic suppression of human lung cancer cells. Drug Deliv. 2019;26 (1):499-508. doi:10.1080/10717544.2019.1606363

52. Wang L, Liu Y, Li W, et al. Selective targeting of gold nanorods at the mitochondria of cancer cells: implications for cancer therapy. Nano Lett. 2011;11(2):772-780. doi:10.1021/nl103992v

53. Zhang J, Chen HB, Zhou T, et al. A PIID-DTBT based semi-conducting polymer dots with broad and strong optical absorption in the visible-light region: highly effective contrast agents for multiscale and multi-spectral photoacoustic imaging. Nano Res. 2017;10(1):64-76. doi:10.1007/s12274-016-1266-8

54. Chen HB, Zhang J, Chang KW, et al. Highly absorbing multispectral near-infrared polymer nanoparticles from one conjugated backbone for photoacoustic imaging and photothermal therapy. Biomaterials. 2017;144:42-52. doi:10.1016/j.biomaterials.2017.08.007

55. Ruttala HB, Ramasamy T, Ruttala RRT, et al. Mitochondria-targeting multi-metallic $\mathrm{ZnCuO}$ nanoparticles and IR780 for efficient photodynamic and photothermal cancer treatments. J Mater Sci Technol. 2021;86:139-150. doi:10.1016/j.jmst.2021.01.035

56. Nikoobakht B, El-Sayed M. Preparation and growth mechanism of gold nanorods (NRs) using seed-mediated growth method. Chem Mater. 2003;15(10):1957-1962. doi:10.1021/cm0207321

57. Huang WT, Chan MH, Chen X, Hsiao M, Liu RS. Theranostic nanobubble encapsulating a plasmon-enhanced upconversion hybrid nanosystem for cancer therapy. Theranostics. 2020;10(2):782-796. doi:10.7150/thno. 38684

58. Englebienne P, Van Hoonacker A, Verhas M, Khlebtsov NG. Advances in high-throughput screening: biomolecular interaction monitoring in real-time with colloidal metal nanoparticles. Comb Chem High Throughput Screen. 2003;6(8):777-787. doi:10.2174/ 138620703771826955

59. Siegel J, Krajcar R, Kolska Z, Hnatowicz V, Svorcik V. Annealing of gold nanostructures sputtered on polytetrafluoroethylene. Nanoscale Res Lett. 2011;6:588. doi:10.1186/1556-276X-6-588

60. Roper DK, Ahn W, Hoepfner M. Microscale heat transfer transduced by surface plasmon resonant gold nanoparticles. J Phys Chem C Nanomater Interfaces. 2007;111(9):3636-3641. doi:10.1021/ jp064341w

61. Li C, Yang XQ, An J, et al. A near-infrared light-controlled smart nanocarrier with reversible polypeptide-engineered valve for targeted fluorescence-photoacoustic bimodal imaging-guided chemo-photothermal therapy. Theranostics. 2019;9(25):7666-7679. doi: $10.7150 /$ thno. 37047

62. Kim J, Chun SH, Amornkitbamrung L, et al. Gold nanoparticle clusters for the investigation of therapeutic efficiency against prostate cancer under near-infrared irradiation. Nano Converg. 2020;7(1):5. doi:10.1186/s40580-019-0216-Z

63. Xu X, Chong Y, Liu X, et al. Multifunctional nanotheranostic gold nanocages for photoacoustic imaging guided radio/photodynamic/ photothermal synergistic therapy. Acta Biomater. 2019;84:328-338. doi:10.1016/j.actbio.2018.11.043 
64. Wang LM, Sun Q, Wang X, et al. Using hollow carbon nanospheres as a light-induced free radical generator to overcome chemotherapy resistance. J Am Chem Soc. 2015;137(5):1947-1955. doi:10.1021/ ja511560b
65. Xu C, Feng Q, Yang H, et al. A light-triggered mesenchymal stem cell delivery system for photoacoustic imaging and chemo-photothermal therapy of triple negative breast cancer. $A d v$ Sci. 2018;5(10):1800382. doi:10.1002/advs.201800382

\section{Publish your work in this journal}

The International Journal of Nanomedicine is an international, peerreviewed journal focusing on the application of nanotechnology in diagnostics, therapeutics, and drug delivery systems throughout the biomedical field. This journal is indexed on PubMed Central, MedLine, CAS, SciSearch ${ }^{\mathbb{R}}$, Current Contents ${ }^{\mathbb{R}} /$ Clinical Medicine, $^{-}$
Journal Citation Reports/Science Edition, EMBase, Scopus and the Elsevier Bibliographic databases. The manuscript management system is completely online and includes a very quick and fair peer-review system, which is all easy to use. Visit http://www.dovepress.com/ testimonials.php to read real quotes from published authors. 